\title{
Convergence of a finite volume scheme for a system of interacting species with cross-diffusion
}

\author{
José A. Carrillo ${ }^{1}$. Francis Filbet ${ }^{2} \cdot$ Markus Schmidtchen $^{3}$ \\ Received: 6 October 2019 / Revised: 26 March 2020 / Published online: 26 May 2020 \\ (c) The Author(s) 2020
}

\begin{abstract}
In this work we present the convergence of a positivity preserving semi-discrete finite volume scheme for a coupled system of two non-local partial differential equations with cross-diffusion. The key to proving the convergence result is to establish positivity in order to obtain a discrete energy estimate to obtain compactness. We numerically observe the convergence to reference solutions with a first order accuracy in space. Moreover we recover segregated stationary states in spite of the regularising effect of the self-diffusion. However, if the self-diffusion or the cross-diffusion is strong enough, mixing occurs while both densities remain continuous.
\end{abstract}

Mathematics Subject Classification Primary 74S10 - 65M12 - 92C15; Secondary $45 \mathrm{~K} 05 \cdot 92 \mathrm{D} 25 \cdot 47 \mathrm{~N} 60$

\section{Introduction}

In this paper we develop and analyse a numerical scheme for the following non-local interaction system with cross-diffusion and self-diffusion

\footnotetext{
$凶$ José A. Carrillo

carrillo@maths.ox.ac.uk

Francis Filbet

francis.filbet@math.univ-toulouse.fr

Markus Schmidtchen

schmidtchen@1jll.math.upmc.fr

1 Mathematical Institute, University of Oxford, Oxford OX2 6GG, UK

2 Institut de Mathématiques de Toulouse, Université Paul Sabatier, Toulouse, France

3 Laboratoire Jacques-Louis Lions, Sorbonne Université, 4 place Jussieu, 75005 Paris, France
} 


$$
\begin{aligned}
& \frac{\partial \rho}{\partial t}=\frac{\partial}{\partial x}\left(\rho \frac{\partial}{\partial x}\left(W_{11 \star \rho}+W_{12 \star \eta}+v(\rho+\eta)\right)+\frac{\epsilon}{2} \frac{\partial \rho^{2}}{\partial x}\right), \\
& \frac{\partial \eta}{\partial t}=\frac{\partial}{\partial x}\left(\eta \frac{\partial}{\partial x}\left(W_{22} \star \eta+W_{21} \star \rho+v(\rho+\eta)\right)+\frac{\epsilon}{2} \frac{\partial \eta^{2}}{\partial x}\right),
\end{aligned}
$$

governing the evolution of two species $\rho$ and $\eta$ on an interval $(a, b) \subset \mathbb{R}$ for $t \in[0, T)$. The system is equipped with nonnegative initial data $\rho^{0}, \eta^{0} \in L_{+}^{1}(a, b) \cap L_{+}^{\infty}(a, b)$. We denote by $m_{1}$ the mass of $\rho_{0}$ and by $m_{2}$ the mass of $\eta_{0}$, respectively,

$$
m_{1}=\int_{a}^{b} \rho_{0}(x) \mathrm{d} x, \text { and } m_{2}=\int_{a}^{b} \eta_{0}(x) \mathrm{d} x .
$$

On the boundary $x=a$ and $b$, we prescribe no-flux boundary conditions

$$
\begin{aligned}
& \rho \frac{\partial}{\partial x}\left(W_{11 \star \rho}+W_{12 \star} \star \eta+v(\rho+\eta)+\epsilon \rho\right)=0, \\
& \eta \frac{\partial}{\partial x}\left(W_{22} \star \eta+W_{21} \star \rho+v(\rho+\eta)+\epsilon \eta\right)=0,
\end{aligned}
$$

such that the total mass of each species is conserved with respect to time $t \geq 0$. While the self-interaction potentials $W_{11}, W_{22} \in C_{b}^{2}(\mathbb{R})$ model the interactions among individuals of the same species (also referred to as intraspecific interactions), the crossinteraction potentials $W_{12}, W_{21} \in C_{b}^{2}(\mathbb{R})$ encode the interactions between individuals belonging to different species, i.e. interspecific interactions. Here $C_{b}^{2}(\mathbb{R})$ denotes the set of twice continuously differentiable functions on $\mathbb{R}$ with bounded derivatives. Notice that the convolutions $W_{i j} \star \psi$, with $\psi$ a density function defined on $[a, b]$, are defined by extending the density $\psi$ by zero outside the interval $[a, b]$. The two positive parameters $\epsilon, v>0$ determine the strengths of the self-diffusion and the cross-diffusion of both species, respectively. Nonlinear diffusion, be it self-diffusion or cross-diffusion, is biologically relevant. As a matter of fact, around the second half of the twentieth century biologists found that the dispersal rate of certain insects depends on the density itself, leading to the nonlinear diffusion terms we incorporated in the model, cf. $[13,32,34,36,37]$. At the same time we would like to stress that the self-diffusion terms are relevant for the convergence analysis below.

It is the interplay between the non-local interactions of both species and their individual and joint size-exclusion, modelled by the non-linear diffusion $[4-6,10,12$, 40], that leads to a large variety of behaviours including complete phase separation or mixing of both densities in both stationary configurations and travelling pulses $[11,19]$.

While their single species counterparts have been studied quite intensively [15, $21,35,41]$ and references therein, related two-species models like the system of our interest, Eq. (1), have only recently gained considerable attention [11,17,19,24,25]. One of the most striking phenomena of these interaction models with cross-diffusion is the possibility of phase separation. Since the seminal papers [5,33] established segregation effects for the first time for the purely diffusive system corresponding to (1) for $W_{i j} \equiv 0, i, j \in\{1,2\}$ and $\epsilon=0$, many generalisations were presented. 
This includes reaction-(cross-)diffusion systems [3,7,17] and references therein, and by adding non-local interactions [2,11,19,24] and references therein. Ref. [24] have established the existence of weak solutions to a class of non-local systems under a strong coercivity assumption on the cross-diffusion also satisfied by system (1).

Typical applications of these non-local models comprise many biological contexts such cell-cell adhesion [16,38,39], for instance, as well as tumour models [26,31], but also the formation of the characteristic stripe patterns of zebrafish can be modelled by these non-local models [42]. Systems of this kind are truly ubiquitous in nature and we remark that 'species' may not only refer to biological species but also to a much wider class of (possibly inanimate) agents such as planets, physical or chemical particles, just to name a few.

Since system (1) is in conservative form a finite volume scheme is a natural choice as a numerical method. This is owing to the fact that, by construction, finite volume schemes are locally conservative: due to the divergence theorem, the change in density on a test cell has to equal the sum of the in-flux and the out-flux of the same cell. There is a huge literature on finite volume schemes, first and foremost [28]. Therein, the authors give a detailed description of the construction of such methods and address convergence issues. Schemes similar to the one proposed in Sect. 2 have been studied in [9] in the case of nonlinear degenerate diffusion equations in any dimension. A similar scheme for a system of two coupled PDEs was proposed in [22]. Later, the authors in [14] generalised the scheme proposed in [9] including both local and non-local drifts. The scheme was then extended to two species in [19]. All the aforementioned schemes have in common that they preserve nonnegativity - a property that is also crucial for our analysis.

Before we define the finite volume scheme we shall present a formal energy estimate for the continuous system. The main difficulty in this paper is to establish positivity and reproducing the continuous energy estimate at the discrete level. The remainder of the introduction is dedicated to presenting the aforementioned energy estimate. Let us consider

$$
\begin{aligned}
\frac{\mathrm{d}}{\mathrm{d} t} \int_{a}^{b} \rho \log \rho \mathrm{d} x & =\int_{a}^{b} \log \rho \frac{\partial \rho}{\partial t} \mathrm{~d} x \\
& =\int_{a}^{b} \log \rho \frac{\partial}{\partial x}\left(\rho \frac{\partial}{\partial x}\left(W_{11 \star}{ }^{b}+W_{12} \star \eta+v(\rho+\eta)+\epsilon \rho\right)\right) \mathrm{d} x \\
& =-\int_{a}^{b} \rho \frac{\partial}{\partial x}\left(W_{11 \star \rho}+W_{12} \star \eta+v(\rho+\eta)+\epsilon \rho\right) \frac{\partial}{\partial x}(\log \rho) \mathrm{d} x,
\end{aligned}
$$

where the second equality holds due to the no-flux boundary conditions. Upon rearranging we get

$$
\begin{aligned}
& \frac{\mathrm{d}}{\mathrm{d} t} \int_{a}^{b} \rho \log \rho \mathrm{d} x+v \int_{a}^{b} \frac{\partial}{\partial x}(\rho+\eta) \frac{\partial \rho}{\partial x} \mathrm{~d} x+\epsilon \int_{a}^{b}\left|\frac{\partial \rho}{\partial x}\right|^{2} \mathrm{~d} x \\
& \quad=-\int_{a}^{b}\left(W_{11^{\prime}}^{\prime} \rho+W_{12}^{\prime} \star \eta\right) \frac{\partial \rho}{\partial x} \mathrm{~d} x .
\end{aligned}
$$


A similar computation for $\eta$ yields

$$
\begin{aligned}
& \frac{\mathrm{d}}{\mathrm{d} t} \int_{a}^{b} \eta \log \eta \mathrm{d} x+v \int_{a}^{b} \frac{\partial}{\partial x}(\rho+\eta) \frac{\partial \eta}{\partial x} \mathrm{~d} x+\epsilon \int_{a}^{b}\left|\frac{\partial \eta}{\partial x}\right|^{2} \mathrm{~d} x \\
& =-\int_{a}^{b}\left(W_{22}^{\prime} \star \eta+W_{21}^{\prime} \star \rho\right) \frac{\partial \eta}{\partial x} \mathrm{~d} x,
\end{aligned}
$$

whence, upon adding both, we obtain

$$
\begin{aligned}
& \frac{\mathrm{d}}{\mathrm{d} t} \int_{a}^{b}[\rho \log \rho+\eta \log \eta] \mathrm{d} x+v \int_{a}^{b}\left|\frac{\partial \sigma}{\partial x}\right|^{2} \mathrm{~d} x \\
& +\epsilon \int_{a}^{b}\left(\left|\frac{\partial \rho}{\partial x}\right|^{2}+\left|\frac{\partial \eta}{\partial x}\right|^{2}\right) \mathrm{d} x=\mathcal{D}_{\rho}+\mathcal{D}_{\eta},
\end{aligned}
$$

where $\sigma=\rho+\eta$ and

$$
\begin{aligned}
& \mathcal{D}_{\rho}:=-\int_{a}^{b}\left(W_{11}^{\prime} \star \rho+W_{12}^{\prime} \star \eta\right) \frac{\partial \rho}{\partial x} \mathrm{~d} x, \\
& \mathcal{D}_{\eta}:=-\int_{a}^{b}\left(W_{22}^{\prime} \star \eta+W_{21}^{\prime} \star \rho\right) \frac{\partial \eta}{\partial x} \mathrm{~d} x,
\end{aligned}
$$

denote the advective parts associated to $\rho$ and $\eta$, respectively. The advective parts can be controlled by using the weighted Young inequality to get

$$
\begin{aligned}
\left|\mathcal{D}_{\rho}\right| & =\left|\int_{a}^{b}\left(W_{11}^{\prime} \star \rho+W_{12}^{\prime} \star \eta\right) \frac{\partial \rho}{\partial x} \mathrm{~d} x\right| \\
& \leq \frac{1}{2 \alpha} \int_{a}^{b}\left|W_{11}^{\prime} \star \rho+W_{12}^{\prime} \star \eta\right|^{2} \mathrm{~d} x+\frac{\alpha}{2} \int_{a}^{b}\left|\frac{\partial \rho}{\partial x}\right|^{2} \mathrm{~d} x,
\end{aligned}
$$

for some $\alpha>0$. In choosing $0<\alpha<\epsilon$ we obtain

$$
\begin{aligned}
& \frac{\mathrm{d}}{\mathrm{d} t} \int_{a}^{b}[\rho \log \rho+\eta \log \eta] \mathrm{d} x+v \int_{a}^{b}\left|\frac{\partial \sigma}{\partial x}\right|^{2} \mathrm{~d} x \\
& \quad+\left(\epsilon-\frac{\alpha}{2}\right) \int_{a}^{b}\left(\left|\frac{\partial \rho}{\partial x}\right|^{2}+\left|\frac{\partial \eta}{\partial x}\right|^{2}\right) \mathrm{d} x \leq \frac{C_{\rho}+C_{\eta}}{2 \alpha},
\end{aligned}
$$

where $C_{\rho}=\left\|W_{11^{\star}}^{\prime} \star \rho+W_{12}^{\prime} \star \eta\right\|_{L^{2}}^{2}$ and $C_{\eta}=\left\|W_{22^{\star}}^{\prime} \star \eta+W_{21}^{\prime} \star \rho\right\|_{L^{2}}^{2}$. From the last line, Eq. (2), we may deduce bounds on the gradient of each species as well as on their sum. As mentioned above the crucial ingredient for this estimate is the positivity of solutions.

The rest of this paper is organised as follows. In the subsequent section we present a semi-discrete finite volume approximation of system (1) and we present the main 
result, Theorem 2.4. Section 3 is dedicated to establishing positivity and to the derivation of a priori estimates. In Sect. 4 we obtain compactness, pass to the limit, and identify the limiting functions as weak solutions to system (1). We conclude the paper with a numerical exploration in Sect. 6. We study the numerical order of accuracy and discuss stationary states and phase segregation phenomena.

\section{Numerical scheme and main result}

In this section we introduce the semi-discrete finite volume scheme for system (1). To begin with, let us introduce our notion of weak solutions.

Definition 2.1 (Weak solutions) A couple of functions $(\rho, \eta) \in L^{2}\left(0, T ; H^{1}(a, b)\right)^{2}$ is a weak solution to system (1) if it satisfies

$$
\begin{aligned}
& -\int_{a}^{b} \rho_{0} \varphi(0, \cdot) \mathrm{d} x \\
& =\int_{0}^{T} \int_{a}^{b}\left[\rho\left(\frac{\partial \varphi}{\partial t}+\left(-v \frac{\partial \sigma}{\partial x}+\frac{\partial V_{1}}{\partial x}\right) \frac{\partial \varphi}{\partial x}\right)+\frac{\epsilon}{2} \rho^{2} \frac{\partial^{2} \varphi}{\partial x^{2}}\right] \mathrm{d} x \mathrm{~d} t
\end{aligned}
$$

and

$$
\begin{aligned}
& -\int_{a}^{b} \eta_{0} \varphi(0, \cdot) \mathrm{d} x \\
& =\int_{0}^{T} \int_{a}^{b}\left[\eta\left(\frac{\partial \varphi}{\partial t}+\left(-v \frac{\partial \sigma}{\partial x}+\frac{\partial V_{2}}{\partial x}\right) \frac{\partial \varphi}{\partial x}\right)+\frac{\epsilon}{2} \eta^{2} \frac{\partial^{2} \varphi}{\partial x^{2}}\right] \mathrm{d} x \mathrm{~d} t
\end{aligned}
$$

respectively, for any $\varphi \in C_{c}^{\infty}([0, T) \times(a, b) ; \mathbb{R})$. Here we have set $V_{k}=-W_{k 1} \star \rho-$ $W_{k 2} \star \eta$, for $k \in\{1,2\}$, and $\sigma=\rho+\eta$, as above.

Notice that the existence of weak solutions to system (1) will follow directly from the convergence of the numerical solution. Indeed, our analysis relies on a compactness argument which does not suppose a priori existence of solution to system (1).

To this end we first define the following space discretisation of the domain.

Definition 2.2 (Space discretisation) To discretise space, we introduce the mesh

$$
\mathcal{T}:=\bigcup_{i \in I} C_{i},
$$

where the control volumes are given by $C_{i}=\left[x_{i-1 / 2}, x_{i+1 / 2}\right)$ for all $i \in I:=$ $\{1, \ldots, N\}$. We assume that the measure of the control volumes are given by $\left|C_{i}\right|=$ $\Delta x_{i}=x_{i+1 / 2}-x_{i-1 / 2}>0$, for all $i \in I$. Note that $x_{1 / 2}=a$, and $x_{N+1 / 2}=b$, cf. (Fig. 1).

We also define $x_{i}=\left(x_{i+1 / 2}+x_{i-1 / 2}\right) / 2$ the centre of cell $C_{i}$ and set $\Delta x_{i+1 / 2}=$ $x_{i+1}-x_{i}$ for $i=1, \ldots, N-1$. We assume that the mesh is regular in the sense that there exists $\xi \in(0,1)$ such that for $h:=\max _{1 \leq i \leq N}\left\{\Delta x_{i}\right\}$ 


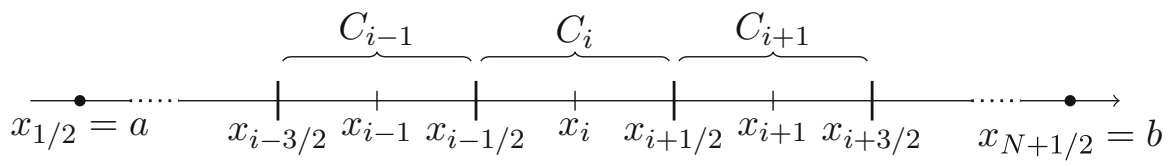

Fig. 1 Space discretisation according to Definition 2.2

$$
\xi h \leq \Delta x_{i} \leq h,
$$

and, as a consequence, $\xi h \leq \Delta x_{i+1 / 2} \leq h$, as well.

On this mesh we shall now define the semi-discrete finite volume approximation of system (1). The discretised initial data are given by the cell averages of the continuous initial data, i.e.

$$
\rho_{i}^{0}:=\frac{1}{\Delta x_{i}} \int_{C_{i}} \rho_{0}(x) \mathrm{d} x, \quad \text { and } \quad \eta_{i}^{0}:=\frac{1}{\Delta x_{i}} \int_{C_{i}} \eta_{0}(x) \mathrm{d} x,
$$

for all $i \in I$. Throughout, we write $\rho_{i}$ (resp. $\eta_{i}$ ) to denote the approximations of the two densities on the $i$-th finite volume cell, $C_{i}$. Next, we introduce the discrete versions of the cross-diffusion and the interaction terms. We set

$$
\begin{aligned}
& \left(V_{1}\right)_{i}:=-\sum_{j=1}^{N} \Delta x_{j}\left(W_{11}^{i-j} \rho_{j}+W_{12}^{i-j} \eta_{j}\right), \\
& \left(V_{2}\right)_{i}:=-\sum_{j=1}^{N} \Delta x_{j}\left(W_{22}^{i-j} \eta_{j}+W_{21}^{i-j} \rho_{j}\right),
\end{aligned}
$$

where

$$
W_{k l}^{i-j}=\frac{1}{\Delta x_{j}} \int_{C_{j}} W_{k l}\left(\left|x_{i}-s\right|\right) \mathrm{d} s,
$$

for $k, l=1,2$, and

$$
U_{i}:=-\left(\rho_{i}+\eta_{i}\right)
$$

for the cross-diffusion term, respectively. Then the scheme reads

$$
\begin{aligned}
\frac{\mathrm{d} \rho_{i}}{\mathrm{~d} t}(t) & =-\frac{\mathcal{F}_{i+1 / 2}(t)-\mathcal{F}_{i-1 / 2}(t)}{\Delta x_{i}}, \\
\frac{\mathrm{d} \eta_{i}}{\mathrm{~d} t}(t) & =-\frac{\mathcal{G}_{i+1 / 2}(t)-\mathcal{G}_{i-1 / 2}(t)}{\Delta x_{i}},
\end{aligned}
$$

for $i \in I$. Here the numerical fluxes are given by 


$$
\begin{aligned}
& \mathcal{F}_{i+1 / 2}(t)=\left[v(\mathrm{~d} U)_{i+1 / 2}^{+}+\left(\mathrm{d} V_{1}\right)_{i+1 / 2}^{+}\right] \rho_{i} \\
& +\left[v(\mathrm{~d} U)_{i+1 / 2}^{-}+\left(\mathrm{d} V_{1}\right)_{i+1 / 2}^{-}\right] \rho_{i+1} \\
& \quad-\frac{\epsilon}{2} \frac{\rho_{i+1}^{2}-\rho_{i}^{2}}{\Delta x_{i+1 / 2}}, \\
& \mathcal{G}_{i+1 / 2}(t)=\left[v(\mathrm{~d} U)_{i+1 / 2}^{+}+\left(\mathrm{d} V_{2}\right)_{i+1 / 2}^{+}\right] \eta_{i} \\
& +\left[v(\mathrm{~d} U)_{i+1 / 2}^{-}+\left(\mathrm{d} V_{2}\right)_{i+1 / 2}^{-}\right] \eta_{i+1} \\
& \quad-\frac{\epsilon}{2} \frac{\eta_{i+1}^{2}-\eta_{i}^{2}}{\Delta x_{i+1 / 2}},
\end{aligned}
$$

for $i=1, \ldots, N-1$, with the numerical no-flux boundary condition

$$
\mathcal{F}_{1 / 2}(t)=\mathcal{F}_{N+1 / 2}(t)=0, \quad \text { and } \quad \mathcal{G}_{1 / 2}(t)=\mathcal{G}_{N+1 / 2}(t)=0,
$$

where we introduced the discrete gradient $\mathrm{d} u_{i+1 / 2}$ as

$$
\mathrm{d} u_{i+1 / 2}:=\frac{u_{i+1}-u_{i}}{\Delta x_{i+1 / 2}}
$$

As usual, we use $(z)^{ \pm}$to denote the positive (resp. negative) part of $z$, i.e.

$$
(z)^{+}:=\max (z, 0), \quad \text { and }(z)^{-}:=\min (z, 0) .
$$

At this stage, the numerical flux (9b) may look strange since

- the cross-diffusion term is approximated as a convective term using that

$$
\frac{\partial}{\partial x}\left(\rho \frac{\partial}{\partial x}(\rho+\eta)\right)=\frac{\partial}{\partial x}\left(\rho \frac{\partial \sigma}{\partial x}\right)
$$

with $\sigma=\rho+\eta$ and $\frac{\partial \sigma}{\partial x}$ is considered as a velocity field. This treatment has already been used in [9] and allows to preserve the positivity of both discrete densities $(\rho, \eta)$ (see Lemma 3.1), which is crucial for the convergence analysis.

- In this new formulation, the velocity field is split in two parts both treated by an upwind scheme. One part comes from the cross-diffusion part, and the second one comes from the non-local interaction fields. This splitting is crucial to recovering a consistent dissipative term for the discrete energy estimate corresponding to Eq. (2).

Definition 2.3 (Piecewise constant approximation) For a given mesh $\mathcal{T}_{h}$ we define the approximate solution to system (1) by

$$
\rho_{h}(t, x):=\rho_{i}(t), \quad \text { and } \quad \eta_{h}(t, x):=\eta_{i}(t)
$$


for all $(t, x) \in[0, T] \times C_{i}$, with $i=1, \ldots, N$. Moreover, we define the following approximations of the gradients

$$
\mathrm{d} \rho_{h}(t, x)=\frac{\rho_{i+1}-\rho_{i}}{\Delta x_{i+1 / 2}}, \quad \text { and } \quad \mathrm{d} \eta_{h}(t, x)=\frac{\eta_{i+1}-\eta_{i}}{\Delta x_{i+1 / 2}}
$$

for $(t, x) \in[0, T) \times\left[x_{i}, x_{i+1}\right)$, for $i=1, \ldots, N-1$. Furthermore, in order to define $\mathrm{d} \rho_{h}$ and $\mathrm{d} \eta_{h}$ on the whole interval $(a, b)$ we set them to zero on $\left(a, x_{1}\right)$ and $\left(x_{N}, b\right)$.

Notice that the discrete gradients $\left(\mathrm{d} \rho_{h}, \mathrm{~d} \eta_{h}\right)$ are piecewise constant just like $\left(\rho_{h}, \eta_{h}\right)$ however not on the same partition of the interval $(a, b)$. In a similar fashion we define the piecewise constant interpolation of the discrete advection fields, i.e.,

$$
\mathrm{d} V_{k, h}(x)=\left(\mathrm{d} V_{k}\right)_{i+1 / 2}
$$

for all $x \in\left[x_{i}, x_{i+1}\right)$, for $i=1, \ldots, N-1$, and zero at the boundary.

We have set out all definitions necessary to formulate the convergence of the numerical scheme (9).

Theorem 2.4 (Convergence to a weak solution) Let $\rho_{0}, \eta_{0} \in L_{+}^{1}(a, b) \cap L_{+}^{\infty}(a, b)$ be some initial data and $Q_{T}:=(0, T) \times(a, b)$. Then,

(i) there exists a nonnegative approximate solution $\left(\rho_{h}, \eta_{h}\right)$ in the sense of Definition 2.3;

(ii) up to a subsequence, this approximate solution converges strongly in $L^{2}\left(Q_{T}\right)$ to $(\rho, \eta) \in L^{2}\left(Q_{T}\right)$, where $(\rho, \eta)$ is a weak solution as in Definition 2.1. Furthermore we have $\rho, \eta \in L^{2}\left(0, T ; H^{1}(a, b)\right)$;

(iii) as a consequence system (1) has a weak solution.

\section{A priori estimates}

This section is dedicated to deriving a priori estimates for our system. In order to do so we require the positivity of approximate solutions and their conservation of mass, respectively. The following lemma guarantees these properties.

Lemma 3.1 (Existence of nonnegative solutions and conservation of mass) Assume that the initial data $\left(\rho_{0}, \eta_{0}\right)$ are non-negative. Then there exists a unique nonnegative approximate solution $\left(\rho_{h}, \eta_{h}\right)_{h>0}$ to the scheme (9a)-(9c). Furthermore, the finite volume scheme conserves the initial mass of both densities.

Proof On the one hand we notice that the right-hand side of (9a)-(9b) is locally Lipschitz with respect to $\left(\rho_{i}, \eta_{i}\right)_{1 \leq i \leq N}$. Hence, we may apply the Cauchy-Lipschitz theorem to obtain a unique continuously differentiable local-in-time solution.

On the other hand to prove that this solution is global in time, we show the nonnegativity of the solution together with the conservation of mass and argue by contradiction. 
On a given mesh, let some initial data, $\rho_{i}(0), \eta_{i}(0) \geq 0$, be given for $i=1, \ldots N$. We rewrite the scheme in the following way.

$$
\frac{\mathrm{d} \rho_{i}}{\mathrm{~d} t}(t)=-\frac{\mathcal{F}_{i+1 / 2}-\mathcal{F}_{i-1 / 2}}{\Delta x_{i}}=\frac{1}{\Delta x_{i}}\left(A_{i} \rho_{i}+B_{i} \rho_{i+1}+C_{i} \rho_{i-1}\right),
$$

where

$$
\begin{aligned}
A_{i}= & v(\mathrm{~d} U)_{i-1 / 2}^{-}+\left(\mathrm{d} V_{1}\right)_{i-1 / 2}^{-}-v(\mathrm{~d} U)_{i+1 / 2}^{+} \\
& -\left(\mathrm{d} V_{1}\right)_{i+1 / 2}^{+}-\frac{\epsilon}{2}\left(\frac{\rho_{i}}{\Delta x_{i+1 / 2}}+\frac{\rho_{i}}{\Delta x_{i-1 / 2}}\right), \\
B_{i}= & -v(\mathrm{~d} U)_{i+1 / 2}^{-}-\left(\mathrm{d} V_{1}\right)_{i+1 / 2}^{-}+\epsilon \frac{\rho_{i+1}}{2 \Delta x_{i+1 / 2}}, \\
C_{i}= & v(\mathrm{~d} U)_{i-1 / 2}^{+}+\left(\mathrm{d} V_{1}\right)_{i-1 / 2}^{+}+\epsilon \frac{\rho_{i-1}}{2 \Delta x_{i-1 / 2}} .
\end{aligned}
$$

Then let $t^{\star} \geq 0$ be the maximal time for all densities to remain nonnegative, i.e.

$$
t^{\star}=\sup \left\{t \geq 0 \mid \rho_{i}(s) \geq 0 \text {, for all } s \in[0, t] \text {, and } i=1, \ldots, N\right\} \text {. }
$$

If $t^{\star}<\infty$, then there exists a nonincreasing sequence $\left(t_{k}\right)_{k \in \mathbb{N}}$ such that $t_{k}>t^{\star}$, $t_{k} \rightarrow t^{\star}$ as $k \rightarrow \infty$ and there exists $i_{k} \in\{1, \ldots, N\}$ verifying

$$
\rho_{i_{k}}\left(t_{k}\right)<0, \quad \forall k \in \mathbb{N}
$$

Since the index $i_{k}$ takes a finite number of integer values, we can extract a nonincreasing subsequence of $\left(t_{k}\right)_{k \in \mathbb{N}}$ still labeled in the same manner such that there exists an index $j_{0} \in\{1, \ldots, N\}$ and

$$
\rho_{j_{0}}\left(t_{k}\right)<0, \quad \forall k \in \mathbb{N}
$$

where $t_{k} \rightarrow t^{\star}$, as $k$ goes to infinity.

Also note by continuity of $\left(\rho_{i}\right)_{1 \leq i \leq N}$, we have that $\rho_{i}\left(t^{\star}\right) \geq 0$ for any $i \in$ $\{1, \ldots, N\}$.

By the above computation, Eq. (10), we see that, if $\rho_{j_{0}+1}\left(t^{\star}\right)>0$ or respectively $\rho_{j_{0}-1}\left(t^{\star}\right)>0$, then either $B_{j_{0}}\left(t^{\star}\right)>0$ or respectively $C_{j_{0}}\left(t^{\star}\right)>0$ and

$$
\begin{aligned}
\frac{\mathrm{d} \rho_{j_{0}}}{\mathrm{~d} t}\left(t^{\star}\right) & =\frac{1}{\Delta x_{j_{0}}}\left(A_{j_{0}} \rho_{j_{0}}\left(t^{\star}\right)+B_{j_{0}} \rho_{j_{0}+1}\left(t^{\star}\right)+C_{j_{0}} \rho_{j_{0}-1}\left(t^{\star}\right)\right) \\
& =\frac{1}{\Delta x_{j_{0}}}\left(B_{j_{0}} \rho_{j_{0}+1}\left(t^{\star}\right)+C_{j_{0}} \rho_{j_{0}-1}\left(t^{\star}\right)\right)>0
\end{aligned}
$$

hence there exists $\tau>0$ such that for any $t \in\left[t^{\star}, t^{\star}+\tau\right)$, we have $\rho_{j_{0}}(t)>\rho_{j_{0}}\left(t^{\star}\right)=$ 0 , which cannot occur since $\rho_{j_{0}}$ is continuous and for $t_{k}>t^{\star}, \rho_{j_{0}}\left(t_{k}\right)<0$ for any $k \in \mathbb{N}$ with $t_{k} \rightarrow t^{\star}$ when $k$ goes to infinity. 
If $\rho_{j_{0}-1}\left(t^{\star}\right)=\rho_{j_{0}}\left(t^{\star}\right)=\rho_{j_{0}+1}\left(t^{\star}\right)=0$ then by uniqueness of the solution, we have that $\rho_{j_{0}} \equiv 0$ for $t \geq t^{\star}$, which contradicts again that $\rho_{j_{0}}\left(t_{k}\right)<0$ for any $k \in \mathbb{N}$ large enough.

Finally we get the conservation of mass,

$$
\begin{aligned}
\frac{\mathrm{d}}{\mathrm{d} t} \int_{a}^{b} \rho_{h}(t, x) \mathrm{d} x & =\sum_{i=1}^{N} \Delta x_{i} \frac{\mathrm{d}}{\mathrm{d} t} \rho_{i} \\
& =\sum_{i=1}^{N} \Delta x_{i} \frac{\mathcal{F}_{i+1 / 2}-\mathcal{F}_{i-1 / 2}}{\Delta x_{i}}=\mathcal{F}_{N+1 / 2}-\mathcal{F}_{1 / 2}=0,
\end{aligned}
$$

by the no-flux condition. Analogously, the second species remains nonnegative and its mass is conserved as well. As a consequence of the control of the $L^{1}$-norm of $\left(\rho_{h}, \eta_{h}\right)$ we can extend the local solution to a global, nonnegative solution.

Now, we are ready to study the evolution of the energy of the system on the semidiscrete level. The remaining part of this section is dedicated to proving the following lemma - an estimate similar to (2) for the semi-discrete scheme (9).

Lemma 3.2 (Energy control) Consider a solution of the semi-discrete scheme (9a)(9b). Then we have

$$
\begin{aligned}
& \frac{\mathrm{d}}{\mathrm{d} t} \sum_{i=1}^{N} \Delta x_{i}\left[\rho_{i} \log \rho_{i}+\eta_{i} \log \eta_{i}\right]+\sum_{i=1}^{N-1} \Delta x_{i+1 / 2}\left[v\left|\mathrm{~d} U_{i+1 / 2}\right|^{2}\right. \\
& \left.\quad+\frac{\epsilon}{4}\left(\left|\mathrm{~d} \rho_{i+1 / 2}\right|^{2}+\left|\mathrm{d} \eta_{i+1 / 2}\right|^{2}\right)\right] \leq C_{\epsilon},
\end{aligned}
$$

where the constant $C_{\epsilon}>0$ is given by

$$
C_{\epsilon}=\frac{(b-a)}{\epsilon}\left(\left(\left\|W_{11}^{\prime}\right\|_{L^{\infty}}+\left\|W_{21}^{\prime}\right\|_{L^{\infty}}\right)^{2} m_{1}^{2}+\left(\left\|W_{12}^{\prime}\right\|_{L^{\infty}}+\left\|W_{22}^{\prime}\right\|_{L^{\infty}}\right)^{2} m_{2}^{2}\right) .
$$

Proof Upon using the scheme, Eq. (9a), we get

$$
\frac{\mathrm{d}}{\mathrm{d} t} \sum_{i=1}^{N} \Delta x_{i} \rho_{i} \log \rho_{i}=-\sum_{i=1}^{N}\left(\mathcal{F}_{i+1 / 2}-\mathcal{F}_{i-1 / 2}\right) \log \rho_{i},
$$

due to the conservation of mass, ensured by Eq. (9c). By discrete integration by parts and the no-flux condition, Eq. (9c), we obtain

$$
\frac{\mathrm{d}}{\mathrm{d} t} \sum_{i=1}^{N} \Delta x_{i} \rho_{i} \log \rho_{i}=\sum_{i=1}^{N-1} \Delta x_{i+1 / 2} \mathcal{F}_{i+1 / 2} \operatorname{d} \log \rho_{i+1 / 2}
$$




$$
\begin{aligned}
= & v \sum_{i=1}^{N-1} \Delta x_{i+1 / 2}\left((\mathrm{~d} U)_{i+1 / 2}^{+} \rho_{i}+(\mathrm{d} U)_{i+1 / 2}^{-} \rho_{i+1}\right) \mathrm{d} \log \rho_{i+1 / 2} \\
& +\sum_{i=1}^{N-1} \Delta x_{i+1 / 2}\left(\left(\mathrm{~d} V_{1}\right)_{i+1 / 2}^{+} \rho_{i}+\left(\mathrm{d} V_{1}\right)_{i+1 / 2}^{-} \rho_{i+1}\right) \mathrm{d} \log \rho_{i+1 / 2}, \\
& -\frac{\epsilon}{2} \sum_{i=1}^{N-1}\left(\rho_{i+1}^{2}-\rho_{i}^{2}\right) \operatorname{dlog} \rho_{i+1 / 2},
\end{aligned}
$$

where, in the last equality, we substituted the definition of the numerical flux, Eq. (9b). Let us define

$$
\tilde{\rho}_{i+1 / 2}:= \begin{cases}\frac{\rho_{i+1}-\rho_{i}}{\log \rho_{i+1}-\log \rho_{i}}, & \text { if } \rho_{i} \neq \rho_{i+1} \\ \frac{\rho_{i}+\rho_{i+1}}{2}, & \text { else, }\end{cases}
$$

for $i \in\{1, \ldots, N-1\}$, and note that then $\tilde{\rho}_{i+1 / 2} \in\left[\rho_{i}, \rho_{i+1}\right]$ by concavity of the $\log$. Here, and throughout, we use the shorthand notation $[x, y]:=[\min (x, y), \max (x, y)]$. Reordering the terms, we obtain

$$
\begin{aligned}
& \frac{\mathrm{d}}{\mathrm{d} t} \sum_{i=1}^{N} \Delta x_{i} \rho_{i} \log \rho_{i}-\sum_{i=1}^{N-1} \Delta x_{i+1 / 2}\left[v \mathrm{~d} U_{i+1 / 2} \tilde{\rho}_{i+1 / 2}-\frac{\epsilon}{2} \mathrm{~d} \rho_{i+1 / 2}^{2}\right] \mathrm{d} \log \rho_{i+1 / 2} \\
& =v \sum_{i=1}^{N-1} \Delta x_{i+1 / 2}\left((\mathrm{~d} U)_{i+1 / 2}^{+}\left(\rho_{i}-\tilde{\rho}_{i+1 / 2}\right)\right. \\
& \left.\quad+(\mathrm{d} U)_{i+1 / 2}^{-}\left(\rho_{i+1}-\tilde{\rho}_{i+1 / 2}\right)\right) \mathrm{d} \log \rho_{i+1 / 2} \\
& \quad+\sum_{i=1}^{N-1} \Delta x_{i+1 / 2}\left(\left(\mathrm{~d} V_{1}\right)_{i+1 / 2}^{+}\left(\rho_{i}-\tilde{\rho}_{i+1 / 2}\right)\right. \\
& \left.\quad+\left(\mathrm{d} V_{1}\right)_{i+1 / 2}^{-}\left(\rho_{i+1}-\tilde{\rho}_{i+1 / 2}\right)\right) \mathrm{d} \log \rho_{i+1 / 2} \\
& \quad+\sum_{i=1}^{N-1} \Delta x_{i+1 / 2} \tilde{\rho}_{i+1 / 2} \mathrm{~d} V_{1, i+1 / 2} \mathrm{~d} \log \rho_{i+1 / 2}
\end{aligned}
$$

Thus, using $\tilde{\rho}_{i+1 / 2} \in\left[\rho_{i}, \rho_{i+1}\right]$ and the monotonicity of $\log$, we note that

$$
\begin{aligned}
& \left(\rho_{i}-\tilde{\rho}_{i+1 / 2}\right) \operatorname{d} \log \rho_{i+1 / 2}\left(v(\mathrm{~d} U)_{i+1 / 2}^{+}+\left(\mathrm{d} V_{1}\right)_{i+1 / 2}^{+}\right) \leq 0, \\
& \left(\rho_{i+1}-\tilde{\rho}_{i+1 / 2}\right) \operatorname{d} \log \rho_{i+1 / 2}\left(v(\mathrm{~d} U)_{i+1 / 2}^{-}+\left(\mathrm{d} V_{1}\right)_{i+1 / 2}^{-}\right) \leq 0 .
\end{aligned}
$$


This is easy to see, for, if $\rho_{i}=\rho_{i+1}$, we observe $\operatorname{dlog} \rho_{i+1 / 2}=0$ and Eqs. (14) hold with equality. In the case of $\rho_{i}<\rho_{i+1}$ we observe

$$
\underbrace{\left(\rho_{i}-\tilde{\rho}_{i+1 / 2}\right)}_{\leq 0} \underbrace{\mathrm{d} \log \rho_{i+1 / 2}}_{\geq 0} \underbrace{\left(v(\mathrm{~d} U)_{i+1 / 2}^{+}+\left(\mathrm{d} V_{1}\right)_{i+1 / 2}^{+}\right)}_{\geq 0} \leq 0,
$$

while, for $\rho_{i}>\rho_{i+1}$ there also holds

$$
\underbrace{\left(\rho_{i}-\tilde{\rho}_{i+1 / 2}\right)}_{\geq 0} \underbrace{\operatorname{dlog} \rho_{i+1 / 2}}_{\leq 0} \underbrace{\left(v(\mathrm{~d} U)_{i+1 / 2}^{+}+\left(\mathrm{d} V_{1}\right)_{i+1 / 2}^{+}\right)}_{\geq 0} \leq 0,
$$

whence we infer the inequality. The same argument can be applied in order to obtain the second line of Eq. (14). Thus we may infer from Eq. (13) that

$$
\begin{aligned}
& \frac{\mathrm{d}}{\mathrm{d} t} \sum_{i=1}^{N} \Delta x_{i} \rho_{i} \log \rho_{i}-\sum_{i=1}^{N-1} \Delta x_{i+1 / 2}\left(v \tilde{\rho}_{i+1 / 2} \mathrm{~d} U_{i+1 / 2}-\frac{\epsilon}{2} \mathrm{~d} \rho_{i+1 / 2}^{2}\right) \mathrm{d} \log \rho_{i+1 / 2} \\
& \quad \leq \sum_{i=1}^{N-1} \Delta x_{i+1 / 2} \tilde{\rho}_{i+1 / 2} \mathrm{~d} \log \rho_{i+1 / 2} \mathrm{~d} V_{1, i+1 / 2} .
\end{aligned}
$$

Note that the definition of $\tilde{\rho}_{i+1 / 2}$ in Eq. (12), is consistent with the case $\rho_{i}=\rho_{i+1}$ and there holds

$$
\tilde{\rho}_{i+1 / 2} \mathrm{~d} \log \rho_{i+1 / 2}=\mathrm{d} \rho_{i+1 / 2}
$$

whence we get

$$
\begin{aligned}
& \frac{\mathrm{d}}{\mathrm{d} t} \sum_{i=1}^{N} \Delta x_{i} \rho_{i} \log \rho_{i}-v \sum_{i=1}^{N-1} \Delta x_{i+1 / 2} \mathrm{~d} \rho_{i+1 / 2} \mathrm{~d} U_{i+1 / 2} \\
& \quad+\frac{\epsilon}{2} \sum_{i=1}^{N-1} \Delta x_{i+1 / 2} \mathrm{~d} \rho_{i+1 / 2}^{2} \operatorname{dlog} \rho_{i+1 / 2} \leq \sum_{i=1}^{N-1} \Delta x_{i+1 / 2} \mathrm{~d} \rho_{i+1 / 2} \mathrm{~d} V_{1, i+1 / 2} .
\end{aligned}
$$

Furthermore, we notice that

$$
\frac{1}{2} \mathrm{~d} \rho_{i+1 / 2}^{2} \mathrm{~d} \log \rho_{i+1 / 2}=\frac{\rho_{i+1}+\rho_{i}}{2 \tilde{\rho}_{i+1 / 2}}\left|\mathrm{~d} \rho_{i+1 / 2}\right|^{2} \geq \frac{1}{2}\left|\mathrm{~d} \rho_{i+1 / 2}\right|^{2},
$$


where we employed Eq. (12). Hence we have

$$
\begin{aligned}
& \frac{\mathrm{d}}{\mathrm{d} t} \sum_{i=1}^{N} \Delta x_{i} \rho_{i} \log \rho_{i}-v \sum_{i=1}^{N-1} \Delta x_{i+1 / 2} \mathrm{~d} \rho_{i+1 / 2} \mathrm{~d} U_{i+1 / 2} \\
& \quad+\frac{\epsilon}{2} \sum_{i=1}^{N-1} \Delta x_{i+1 / 2}\left|\mathrm{~d} \rho_{i+1 / 2}\right|^{2} \leq \sum_{i=1}^{N-1} \Delta x_{i+1 / 2} \mathrm{~d} \rho_{i+1 / 2} \mathrm{~d} V_{1, i+1 / 2} .
\end{aligned}
$$

A similar computation can be applied to the second species, which yields

$$
\begin{aligned}
& \frac{\mathrm{d}}{\mathrm{d} t} \sum_{i=1}^{N} \Delta x_{i} \eta_{i} \log \eta_{i}-v \sum_{i=1}^{N-1} \Delta x_{i+1 / 2} \mathrm{~d} \eta_{i+1 / 2} \mathrm{~d} U_{i+1 / 2} \\
& \quad+\frac{\epsilon}{2} \sum_{i=1}^{N-1} \Delta x_{i+1 / 2}\left|\mathrm{~d} \eta_{i+1 / 2}\right|^{2} \leq \sum_{i=1}^{N-1} \Delta x_{i+1 / 2} \mathrm{~d} \eta_{i+1 / 2} \mathrm{~d} V_{2, i+1 / 2}
\end{aligned}
$$

Upon adding up Eqs. (15) and (16), we obtain

$$
\begin{aligned}
& \frac{\mathrm{d}}{\mathrm{d} t} \sum_{i=1}^{N} \Delta x_{i}\left[\rho_{i} \log \rho_{i}+\eta_{i} \log \eta_{i}\right]-v \sum_{i=1}^{N-1} \Delta x_{i+1 / 2} \mathrm{~d} U_{i+1 / 2} \mathrm{~d}(\rho+\eta)_{i+1 / 2} \\
& \quad+\frac{\epsilon}{2} \sum_{i=1}^{N-1} \Delta x_{i+1 / 2}\left(\left|\mathrm{~d} \rho_{i+1 / 2}\right|^{2}+\left|\mathrm{d} \eta_{i+1 / 2}\right|^{2}\right) \leq \mathcal{R}_{h}
\end{aligned}
$$

where $\mathcal{R}_{h}$ is given by

$$
\mathcal{R}_{h}=\sum_{i=1}^{N-1} \Delta x_{i+1 / 2}\left[\mathrm{~d} V_{1, i+1 / 2} \mathrm{~d} \rho_{i+1 / 2}+\mathrm{d} V_{2, i+1 / 2} \mathrm{~d} \eta_{i+1 / 2}\right]
$$

Finally we notice that

$$
\begin{aligned}
\mathcal{R}_{h} \leq & \frac{1}{2} \sum_{i=1}^{N-1} \Delta x_{i+1 / 2}\left[\frac{\left|\mathrm{d} V_{1, i+1 / 2}\right|^{2}}{\alpha}+\alpha\left|\mathrm{d} \rho_{i+1 / 2}\right|^{2}\right. \\
& \left.+\frac{\left|\mathrm{d} V_{2, i+1 / 2}\right|^{2}}{\alpha}+\alpha\left|\mathrm{d} \eta_{i+1 / 2}\right|^{2}\right]
\end{aligned}
$$

for any $\alpha>0$, by Young's inequality. Observing, that for $k=1,2$,

$$
\left|\mathrm{d} V_{k, i+1 / 2}\right| \leq\left|\sum_{j=1}^{N-1} \rho_{j} \int_{C_{j}} \frac{W_{k 1}\left(x_{i+1}-y\right)-W_{k 1}\left(x_{i}-y\right)}{\Delta x_{i+1 / 2}} \mathrm{~d} y\right|
$$




$$
\begin{aligned}
& +\left|\sum_{j=1}^{N-1} \eta_{j} \int_{C_{j}} \frac{W_{k 2}\left(x_{i+1}-y\right)-W_{k 2}\left(x_{i}-y\right)}{\Delta x_{i+1 / 2}} \mathrm{~d} y\right| \\
& \leq\left\|W_{k 1}^{\prime}\right\|_{L^{\infty}} m_{1}+\left\|W_{k 2}^{\prime}\right\|_{L^{\infty} m_{2}}
\end{aligned}
$$

and by conservation of positivity and mass, it gives for $k=1,2$,

$$
\frac{1}{2 \alpha} \sum_{i=1}^{N-1} \Delta x_{i+1 / 2}\left|\mathrm{~d} V_{k, i+1 / 2}\right|^{2} \leq \frac{(b-a)}{2 \alpha}\left(m_{1}\left\|W_{k 1}^{\prime}\right\|_{L^{\infty}}+m_{2}\left\|W_{k 2}^{\prime}\right\|_{L^{\infty}}\right)^{2}
$$

Thus Eq. (18) becomes

$$
\mathcal{R}_{h} \leq \frac{\alpha}{2} \sum_{i=1}^{N-1} \Delta x_{i+1 / 2}\left(\left|\mathrm{~d} \rho_{i+1 / 2}\right|^{2}+\left|\mathrm{d} \eta_{i+1 / 2}\right|^{2}\right)+C_{2 \alpha}
$$

where $C_{2 \alpha}$ is given in Eq. (11). Finally, substituting the latter estimate into Eq. (17), we obtain, upon using Eq. (8),

$$
\begin{aligned}
& \frac{\mathrm{d}}{\mathrm{d} t} \sum_{i=1}^{N} \Delta x_{i}\left[\rho_{i} \log \rho_{i}+\eta_{i} \log \eta_{i}\right]+v \sum_{i=1}^{N-1} \Delta x_{i+1 / 2}\left|\mathrm{~d} U_{i+1 / 2}\right|^{2} \\
& \quad+\frac{\epsilon-\alpha}{2} \sum_{i=1}^{N-1} \Delta x_{i+1 / 2}\left(\left|\mathrm{~d} \rho_{i+1 / 2}\right|^{2}+\left|\mathrm{d} \eta_{i+1 / 2}\right|^{2}\right) \leq C_{2 \alpha},
\end{aligned}
$$

for any solution $\left(\rho_{i}\right)_{i \in I},\left(\eta_{i}\right)_{i \in I}$ of the semi-discrete scheme (9). Hence choosing $\alpha=\epsilon / 2$ concludes the proof.

Corollary 3.3 (A priori bounds) Let $\left(\rho_{i}\right)_{i \in I},\left(\eta_{i}\right)_{i \in I}$ be solutions of the semi-discrete scheme (9). Then there exists a constant $C>0$ such that

$$
\int_{0}^{T} \sum_{i=1}^{N-1} \Delta x_{i+1 / 2}\left(\frac{\epsilon}{4}\left|\mathrm{~d} \rho_{i+1 / 2}\right|^{2}+\frac{\epsilon}{4}\left|\mathrm{~d} \eta_{i+1 / 2}\right|^{2}+v\left|\mathrm{~d} U_{i+1 / 2}\right|^{2}\right) \mathrm{d} t \leq C .
$$

Proof Using the fact that $x \log x \geq-\log (e) / e$, i.e. $x \log x$ is bounded from below, yields

$$
\sum_{i \in I} \Delta x_{i}\left[\rho_{i} \log \rho_{i}+\eta_{i} \log \eta_{i}\right](t) \geq-2 \frac{\log (e)}{e}(b-a)=:-C_{1}
$$

Hence the discrete version of the classical entropy functional is bounded from below. Therefore, we integrate the inequality of Lemma 3.2 in time and get

$$
\int_{0}^{T} \sum_{i=1}^{N-1} \Delta x_{i+1 / 2}\left[v\left|\mathrm{~d} U_{i+1 / 2}(t)\right|^{2}+\frac{\epsilon}{4}\left(\left|\mathrm{~d} \rho_{i+1 / 2}(t)\right|^{2}+\left|\mathrm{d} \eta_{i+1 / 2}(t)\right|^{2}\right)\right] \mathrm{d} t
$$




$$
\leq C_{\epsilon} T+C_{1}+\int_{a}^{b} \rho_{0}\left|\log \rho_{0}\right|+\eta_{0}\left|\log \eta_{0}\right| \mathrm{d} x,
$$

which proves the statement.

Thanks to a classical discrete Poincaré inequality [28, Lemma 3.7] and [8], we get uniform $L^{2}$-estimates on the discrete approximation $\left(\rho_{h}, \eta_{h}\right)_{h>0}$.

Lemma 3.4 Let $\left(\rho_{i}\right)_{i \in I},\left(\eta_{i}\right)_{i \in I}$ be the numerical solutions obtained from scheme (9). Then there holds

$$
\left\|\rho_{h}\right\|_{L^{2}\left(Q_{T}\right)}+\left\|\eta_{h}\right\|_{L^{2}\left(Q_{T}\right)} \leq C,
$$

for some constant $C>0$ independent of $h>0$.

\section{Proof of Theorem 2.4}

This section is dedicated to proving compactness of both species, the fluxes, and the regularising porous-medium type diffusion. Upon establishing the compactness result we identify the limits as weak solutions in the sense of Definition 2.1.

First by application of Lemma 3.1, we get existence and uniqueness of a nonnegative approximate solution $\left(\rho_{h}, \eta_{h}\right)$ to $(9 \mathrm{a})-(9 \mathrm{~b})$. Hence the first item of Theorem 2.4 is proven. Now let us investigate the asymptotic $h \rightarrow 0$.

\subsection{Strong compactness of approximate solutions}

We shall now make use of the above estimates in order to obtain strong compactness of both species, $\left(\rho_{h}, \eta_{h}\right)$ in $L^{2}\left(Q_{T}\right)$.

Lemma 4.1 (Strong compactness in $L^{2}\left(Q_{T}\right)$ ) Let $\left(\rho_{h}, \eta_{h}\right)_{h>0}$ be the approximation to system (1) obtained by the semi-discrete scheme (9). Then there exist functions $\rho, \eta \in L^{2}\left(Q_{T}\right)$ such that

$$
\rho_{h} \rightarrow \rho, \text { and } \eta_{h} \rightarrow \eta,
$$

strongly in $L^{2}\left(Q_{T}\right)$, up to a subsequence.

Proof We invoke the compactness criterion by Aubin and Lions [23]. Accordingly, a set $P \subset L^{2}(0, T ; B)$ is relatively compact if $P$ is bounded in $L^{2}(0, T ; X)$ and the set of derivatives $\left\{\partial_{t} \rho \mid \rho \in P\right\}$ is bounded in a third space $L^{1}(0, T ; Y)$, whenever the involved Banach spaces satisfy $X \hookrightarrow \hookrightarrow B \hookrightarrow Y$, i.e. the first embedding is compact and the second one continuous. For our purpose we choose $X:=B V(a, b)$, $B:=L^{2}(a, b)$, and $Y:=H^{-2}(a, b)$. The first embedding is indeed compact, e.g. Ref. [1, Theorem 10.1.4] and the second one is continuous.

In the second step we show the time derivatives are bounded in $L^{1}\left(0, T ; H^{-2}(a, b)\right)$. To this end, let $\varphi \in C_{c}^{\infty}((a, b))$. Throughout, we write $\langle\cdot, \cdot\rangle$ for $\langle\cdot, \cdot\rangle_{H^{-2}, H^{2}}$ for the 
dual pairing. Making use of the scheme, there holds

$$
\left\langle\frac{\mathrm{d} \rho_{h}}{\mathrm{~d} t}, \varphi\right\rangle=\sum_{i=1}^{N} \int_{C_{i}} \frac{\mathrm{d} \rho_{i}}{\mathrm{~d} t} \varphi \mathrm{d} x=-\sum_{i=1}^{N} \frac{\mathcal{F}_{i+1 / 2}-\mathcal{F}_{i-1 / 2}}{\Delta x_{i}} \int_{C_{i}} \varphi \mathrm{d} x
$$

having used the scheme, Eq. (9a). Next we set

$$
\varphi_{i}:=\frac{1}{\Delta x_{i}} \int_{C_{i}} \varphi \mathrm{d} x
$$

perform a discrete integration by parts and use the no-flux boundary conditions, Eq. (9c), to obtain

$$
\left\langle\frac{\mathrm{d} \rho_{h}}{\mathrm{~d} t}, \varphi\right\rangle=\sum_{i=1}^{N-1} \mathcal{F}_{i+1 / 2}\left(\varphi_{i+1}-\varphi_{i}\right)
$$

Using the definition of the numerical flux, Eq. (9b), we get

$$
\begin{aligned}
\left\langle\frac{\mathrm{d} \rho_{h}}{\mathrm{~d} t}, \varphi\right\rangle= & \sum_{i=1}^{N-1}\left[\left(v(\mathrm{~d} U)_{i+1 / 2}^{+}+\left(\mathrm{d} V_{1}\right)_{i+1 / 2}^{+}\right) \rho_{i}\right. \\
& \left.+\left(v(\mathrm{~d} U)_{i+1 / 2}^{-}+\left(\mathrm{d} V_{1}\right)_{i+1 / 2}^{-}\right) \rho_{i+1}\right]\left(\varphi_{i+1}-\varphi_{i}\right) \\
& -\frac{\epsilon}{2} \sum_{i=1}^{N-1} \frac{\rho_{i+1}^{2}-\rho_{i}^{2}}{\Delta x_{i+1 / 2}}\left(\varphi_{i+1}-\varphi_{i}\right) .
\end{aligned}
$$

Let us begin with the self-diffusion part. Using the Cauchy-Schwarz inequality, we estimate the discrete gradient and $\rho$ itself by Corollary 3.3 and Lemma 3.4

$$
\begin{aligned}
& \frac{\epsilon}{2} \int_{0}^{T} \sum_{i=1}^{N-1} \frac{\rho_{i+1}^{2}-\rho_{i}^{2}}{\Delta x_{i+1 / 2}}\left(\varphi_{i+1}-\varphi_{i}\right) \mathrm{d} t \\
& \quad \leq \frac{\epsilon}{2}\left\|\frac{\partial \varphi}{\partial x}\right\|_{L^{\infty}} \int_{0}^{T} \sum_{i=1}^{N-1} \Delta x_{i+1 / 2}\left|\mathrm{~d} \rho_{i+1 / 2}\right|\left(\rho_{i+1}+\rho_{i}\right) \mathrm{d} t \\
& \quad \leq \frac{\epsilon}{2}\left\|\frac{\partial \varphi}{\partial x}\right\|_{L^{\infty}}\left(\int_{0}^{T} \sum_{i=1}^{N-1} \Delta x_{i+1 / 2}\left|\mathrm{~d} \rho_{i+1 / 2}\right|^{2} \mathrm{~d} t\right)^{1 / 2}\left(\int_{0}^{T} \sum_{i=1}^{N} 4 \xi^{-1} \Delta x_{i}\left|\rho_{i}\right|^{2} \mathrm{~d} t\right)^{1 / 2} \\
& \quad \leq C\|\varphi\|_{H^{2}(a, b)},
\end{aligned}
$$

where we used that $\varphi^{\prime} \in H^{1} \subset L^{\infty}$ and the regularity of the mesh, $\xi>0$, cf. Eq. (4). 
Next, we address the cross-diffusion and non-local interactions terms using the same argument. For instance for the cross-diffusive part, we have

$$
\begin{aligned}
& v \int_{0}^{T}\left|\sum_{i=1}^{N-1}\left[(\mathrm{~d} U)_{i+1 / 2}^{+} \rho_{i}+(\mathrm{d} U)_{i+1 / 2}^{-} \rho_{i+1}\right]\right|\left|\varphi_{i+1}-\varphi_{i}\right| \mathrm{d} t \\
& \quad \leq \frac{2 v}{\sqrt{\xi}}\left\|\frac{\partial \varphi}{\partial x}\right\|_{L^{\infty}}\left(\int_{0}^{T} \sum_{i=1}^{N-1} \Delta x_{i+1 / 2}\left|\mathrm{~d} U_{i+1 / 2}\right|^{2} \mathrm{~d} t\right)^{1 / 2}\left(\int_{0}^{T} \sum_{i=1}^{N} \Delta x_{i} \rho_{i}^{2} \mathrm{~d} t\right)^{1 / 2} \\
& \quad \leq C\|\varphi\|_{H^{2}(a, b)},
\end{aligned}
$$

where we used Corollary 3.3 and Lemma 3.4 again. The non-local interaction term is estimated in the same way, thus there holds

$$
\int_{0}^{T}\left|\left\langle\frac{\mathrm{d} \rho_{h}}{\mathrm{~d} t}, \varphi\right\rangle\right| \mathrm{d} t \leq C\|\varphi\|_{H^{2}(a, b)} .
$$

By density of $C_{c}^{\infty}((a, b))$ in $H_{0}^{2}(a, b)$ we may infer the boundedness of $\left(\frac{\mathrm{d} \rho_{h}}{\mathrm{~d} t}\right)_{h>0}$ in $L^{1}\left(0, T ; H^{-2}(a, b)\right)$, which concludes the proof.

From the latter result we can prove the convergence of the discrete advection field $\mathrm{d} V_{1, h}$ and $\mathrm{d} V_{2, h}$ defined as in Definition 2.3.

Lemma 4.2 For any $1 \leq p \leq \infty$ and $k \in\{1,2\}$, the piecewise constant approximation $\mathrm{d} V_{k, h}$ converges strongly in $L^{2}\left(0, T ; L^{2}(a, b)\right)$ to $-\left(W_{k 1}^{\prime} \star \rho+W_{k 2}^{\prime} \star \eta\right)$, where $(\rho, \eta)$ corresponds to the limit obtained in Lemma 4.1.

Proof Let $k \in\{1,2\}$. For each $i=0, \ldots, N-1$, and $x \in\left[x_{i}, x_{i+1}\right)$ we have

$$
\begin{aligned}
\mathrm{d} V_{k, h}(x)= & \left(\mathrm{d} V_{k}\right)_{i+1 / 2}=-\sum_{j=1}^{N} \int_{C_{j}} \frac{W_{k 1}\left(x_{i+1}-y\right)-W_{k 1}\left(x_{i}-y\right)}{\Delta x_{i+1 / 2}} \rho_{j} \mathrm{~d} y \\
& -\sum_{j=1}^{N} \int_{C_{j}} \frac{W_{k 2}\left(x_{i+1}-y\right)-W_{k 2}\left(x_{i}-y\right)}{\Delta x_{i+1 / 2}} \eta_{j} \mathrm{~d} y .
\end{aligned}
$$

We define $V_{k, h}^{\prime}$ and $V_{k}^{\prime}$ as

$$
\left\{\begin{array}{l}
V_{k, h}^{\prime}(x):=-W_{k 1}^{\prime} \star \rho_{h}-W_{k 2}^{\prime} \star \eta_{h}, \\
V_{k}^{\prime}(x):=-W_{k 1}^{\prime} \star \rho-W_{k 2}^{\prime} \star \eta .
\end{array}\right.
$$

On the one hand from the strong convergence of $\left(\rho_{h}, \eta_{h}\right)$ to $(\rho, \eta)$ in $L^{2}\left(0, T ; L^{2}(a, b)\right)$ and the convolution product's properties, we obtain

$$
\left\|V_{k, h}^{\prime}-V_{k}^{\prime}\right\|_{L^{2}\left(0, T ; L^{2}(a, b)\right)} \rightarrow 0, \text { when } h \rightarrow 0
$$


On the other hand, we have for any $x \in\left[x_{i}, x_{i+1}\right)$

$$
\begin{aligned}
& \left|\mathrm{d} V_{k, h}(x)-V_{k, h}^{\prime}(x)\right| \\
& \leq \sum_{j=1}^{N} \int_{C_{j}}\left|\frac{W_{k 1}\left(x_{i+1}-y\right)-W_{k 1}\left(x_{i}-y\right)}{\Delta x_{i+1 / 2}}-W_{k 1}^{\prime}(x-y)\right| \rho_{j} \mathrm{~d} y, \\
& \quad+\sum_{j=1}^{N} \int_{C_{j}}\left|\frac{W_{k 2}\left(x_{i+1}-y\right)-W_{k 2}\left(x_{i}-y\right)}{\Delta x_{i+1 / 2}}-W_{k 2}^{\prime}(x-y)\right| \eta_{j} \mathrm{~d} y, \\
& \leq\left(\left\|W_{k 1}^{\prime \prime}\right\|_{\left.L^{\infty} m_{1}+\left\|W_{k 2}^{\prime \prime}\right\|_{L^{\infty} m_{2}}\right) h,}\right.
\end{aligned}
$$

hence there exists a constant $C>0$ such that

$$
\left|\mathrm{d} V_{k, h}(x)-V_{k, h}^{\prime}(x)\right|^{2} \leq C^{p} h^{2}
$$

Integrating over $x \in\left[x_{i}, x_{i+1}\right)$ and summing over $i \in\{1, \ldots, N-1\}$, we get that

$$
\left\|\mathrm{d} V_{k, h}-V_{k, h}^{\prime}\right\|_{L^{2}\left(0, T ; L^{2}(a, b)\right)} \rightarrow 0, \text { when } h \rightarrow 0
$$

Notice that $\left(x_{1}, x_{N}\right) \subset(a, b)$ where $x_{1} \rightarrow a$ and $x_{N} \rightarrow b$ as $h \rightarrow 0$. From Eqs. (20) and (21) we get that $\left\|\mathrm{d} V_{k, h}-V_{k}^{\prime}\right\|_{L^{2}\left(0, T ; L^{2}(a, b)\right)}$ goes to zero as $h$ tends to zero.

\subsection{Weak compactness for the discrete gradients}

In the previous section we have established the strong $L^{2}$-convergence of both species, $\left(\rho_{h}\right)_{h>0}$ and $\left(\eta_{h}\right)_{h>0}$. However, in order to be able to pass to the limit in the crossdiffusion term $\rho_{h}\left(\mathrm{~d} \rho_{h}+\mathrm{d} \eta_{h}\right)$ we need to establish weak convergence in the discrete gradients in $L^{2}$. This is done in the following proposition.

Proposition 1 (Weak convergence of the derivatives) The discrete spatial derivatives, defined in Definition 2.3, satisfy $\mathrm{d} \beta_{h}$ converges weakly to $\frac{\partial \beta}{\partial x}$ in $L^{2}\left(Q_{T}\right)$ and $\beta \in$ $L^{2}\left(0, T ; H^{1}(a, b)\right)$, where $\beta \in\{\rho, \eta, U\}$

Proof Take $\beta \in\{\rho, \eta, U\}$, hence from Lemma 4.1, we know that $\beta_{h} \rightarrow \beta$ strongly in $L^{2}\left(Q_{T}\right)$. Furthermore, from Corollary 3.3 we also deduce that $\mathrm{d} \beta_{h}$ weakly converges to some function $r \in L^{2}\left(Q_{T}\right)$.

Let us show that $\beta \in L^{2}\left(0, T, H^{1}(a, b)\right)$ and $r=\frac{\partial \beta}{\partial x}$. First, we have for any $t \in[0, T]$ and any $\varphi \in \mathcal{C}_{c}^{\infty}((0, T) \times(a, b))$,

$$
\begin{aligned}
\int_{Q_{T}} \beta_{h}(t) \frac{\partial \varphi}{\partial x} \mathrm{~d} x & =\int_{0}^{T} \sum_{i=1}^{N} \beta_{i}(t)\left[\varphi\left(t, x_{i+1 / 2}\right)-\varphi\left(t, x_{i-1 / 2}\right)\right] \mathrm{d} t \\
& =-\int_{0}^{T} \sum_{i=1}^{N-1} \Delta x_{i+1 / 2} \mathrm{~d} \beta_{i+1 / 2}(t) \varphi\left(t, x_{i+1 / 2}\right) \mathrm{d} t
\end{aligned}
$$


having used discrete integration by parts and the fact that $\varphi$ is compactly supported, i.e. $\varphi\left(t, x_{N+1 / 2}\right)=\varphi\left(t, x_{1 / 2}\right)=0$. Then, by Definition 2.3 on the discrete gradient, we may consider

$$
\begin{aligned}
& \left|\int_{0}^{T} \sum_{i=1}^{N-1} \int_{x_{i}}^{x_{i+1}} \mathrm{~d} \beta_{i+1 / 2} \varphi(t, x) \mathrm{d} x \mathrm{~d} t+\int_{0}^{T} \int_{a}^{b} \beta_{h} \frac{\partial \varphi}{\partial x} \mathrm{~d} x \mathrm{~d} t\right| \\
& \quad \leq \int_{0}^{T} \sum_{i=1}^{N-1} \int_{x_{i}}^{x_{i+1}}\left|\mathrm{~d} \beta_{i+1 / 2}\right|\left|\varphi(t, x)-\varphi\left(t, x_{i+1 / 2}\right)\right| \mathrm{d} x \mathrm{~d} t \\
& \quad \leq\left\|\frac{\partial \varphi}{\partial x}\right\|_{\infty}\left(\int_{0}^{T} \sum_{i=1}^{N-1} \Delta x_{i+1 / 2}\left|\mathrm{~d} \beta_{i+1 / 2}\right|^{2} \mathrm{~d} t\right)^{1 / 2}\left(\int_{0}^{T} \sum_{i=1}^{N-1} \Delta x_{i+1 / 2}^{3} \mathrm{~d} t\right)^{1 / 2} \\
& \quad \leq\left\|\frac{\partial \varphi}{\partial x}\right\|_{\infty} C^{1 / 2} T^{1 / 2} \sqrt{b-a} h,
\end{aligned}
$$

having used the a priori bounds, cf. Corollary 3.3. This yields the statement, when $h \rightarrow 0$, for we have

$$
\int_{0}^{T} \sum_{i=1}^{N-1} \int_{x_{i}}^{x_{i+1}} \mathrm{~d} \beta_{i+1 / 2} \varphi(t, x) \mathrm{d} x \mathrm{~d} t+\int_{0}^{T} \int_{a}^{b} \beta_{h} \frac{\partial \varphi}{\partial x} \mathrm{~d} x \mathrm{~d} t \rightarrow 0
$$

which proves that $\mathrm{d} \beta_{h}$ converges weakly to $\frac{\partial \beta}{\partial x}$, as $h \rightarrow 0$ and thus $\beta \in$ $L^{2}\left(0, T ; H^{1}(a, b)\right)$.

\subsection{Passing to the limit}

We have now garnered all information necessary to prove Theorem 2.4. For brevity we shall only show the convergence result for $\rho$, as it follows for $\eta$ similarly, using the same arguments. Let $\varphi \in C_{c}^{\infty}([0, T) \times(a, b))$ be a test function. We introduce the following notations:

$$
\begin{aligned}
\mathcal{E}_{h} & :=\int_{0}^{T} \int_{a}^{b} \rho_{h} \frac{\partial \varphi}{\partial t} \mathrm{~d} x \mathrm{~d} t+\int_{a}^{b} \rho_{h}(0) \varphi(0) \mathrm{d} x, \\
\mathcal{A}_{h} & :=\int_{0}^{T} \int_{a}^{b} \mathrm{~d} V_{1, h} \rho_{h} \frac{\partial \varphi}{\partial x} \mathrm{~d} x \mathrm{~d} t \\
\mathcal{C}_{h} & :=v \int_{0}^{T} \int_{a}^{b} \mathrm{~d} U_{h} \rho_{h} \frac{\partial \varphi}{\partial x} \mathrm{~d} x \mathrm{~d} t \\
\mathcal{D}_{h} & :=\frac{\epsilon}{2} \int_{0}^{T} \int_{a}^{b} \rho_{h}^{2} \frac{\partial^{2} \varphi}{\partial x^{2}} \mathrm{~d} x \mathrm{~d} t .
\end{aligned}
$$


and

$$
\varepsilon(h):=\mathcal{E}_{h}+\mathcal{A}_{h}+\mathcal{C}_{h}+\mathcal{D}_{h} .
$$

On the other hand, we set

$$
\varphi_{i}(t)=\frac{1}{\Delta x_{i}} \int_{C_{i}} \varphi(t, x) \mathrm{d} x,
$$

and multiply the scheme, Eq. (9a), by the test function and integrate in time and space to get

$$
\mathcal{E}_{h}+\mathcal{A}_{1, h}+\mathcal{C}_{1, h}+\mathcal{D}_{1, h}=0
$$

where

$$
\begin{aligned}
\mathcal{A}_{1, h} & :=\sum_{i=1}^{N-1} \int_{0}^{T} \Delta x_{i+1 / 2}\left[\left(\mathrm{~d} V_{1}\right)_{i+1 / 2}^{+} \rho_{i}+\left(\mathrm{d} V_{1}\right)_{i+1 / 2}^{-} \rho_{i+1}\right] \mathrm{d} \varphi_{i+1 / 2}(t) \mathrm{d} t, \\
\mathcal{C}_{1, h} & :=v \sum_{i=1}^{N-1} \int_{0}^{T} \Delta x_{i+1 / 2}\left[(\mathrm{~d} U)_{i+1 / 2}^{+} \rho_{i}+(\mathrm{d} U)_{i+1 / 2}^{-} \rho_{i+1}\right] \mathrm{d} \varphi_{i+1 / 2}(t) \mathrm{d} t, \\
\mathcal{D}_{1, h} & :=-\frac{\epsilon}{2} \sum_{i=1}^{N-1} \int_{0}^{T}\left[\rho_{i+1}^{2}-\rho_{i}^{2}\right] \mathrm{d} \varphi_{i+1 / 2}(t) \mathrm{d} t
\end{aligned}
$$

When $h$ tends to zero and from the strong convergence of $\left(\rho_{h}, \eta_{h}\right)_{h>0}$ to $(\rho, \eta)$ in $L^{2}\left(Q_{T}\right)$, the strong convergence of $\left(\mathrm{d} V_{k, h}\right)_{h>0}$ to $V_{k}^{\prime}$ in $L^{2}\left(Q_{T}\right)$ and the weak convergence of the discrete gradient $\left(\mathrm{d} U_{h}\right)_{h>0}$ to $-\frac{\partial \sigma}{\partial x}$ in $L^{2}\left(Q_{T}\right)$, it is easy to see that

$$
\begin{aligned}
\varepsilon(h) & \rightarrow \int_{0}^{T} \int_{a}^{b}\left\{\rho\left[\frac{\partial \varphi}{\partial t}+\left(\frac{\partial V_{1}}{\partial x}-v \frac{\partial}{\partial x}(\rho+\eta)\right) \frac{\partial \varphi}{\partial x}\right]+\frac{\epsilon}{2} \rho^{2} \frac{\partial^{2} \varphi}{\partial x^{2}}\right\} \mathrm{d} x \mathrm{~d} t \\
& +\int_{a}^{b} \rho(0) \varphi(0) \mathrm{d} x
\end{aligned}
$$

when $h \rightarrow 0$. Therefore it suffices to prove that $\varepsilon(h) \rightarrow 0$, as $h$ goes to zero, which will be achieved by proving that $\mathcal{A}_{h}-\mathcal{A}_{1, h}, \mathcal{C}_{h}-\mathcal{C}_{1, h}$ and $\mathcal{D}_{h}-\mathcal{D}_{1, h}$ vanish in the limit $h \rightarrow 0$.

The self-diffusion part $\mathcal{D}_{h}-\mathcal{D}_{1, h}$

On the one hand, after a simple integration we get

$$
\mathcal{D}_{h}=\frac{\epsilon}{2} \sum_{i=1}^{N} \int_{0}^{T} \rho_{i}^{2}(t)\left[\frac{\partial \varphi}{\partial x}\left(t, x_{i+1 / 2}\right)-\frac{\partial \varphi}{\partial x}\left(t, x_{i-1 / 2}\right)\right] \mathrm{d} t
$$




$$
=-\frac{\epsilon}{2} \sum_{i=1}^{N-1} \int_{0}^{T}\left[\rho_{i+1}^{2}(t)-\rho_{i}^{2}(t)\right] \frac{\partial \varphi}{\partial x}\left(t, x_{i+1 / 2}\right) \mathrm{d} t .
$$

Hence, we have

$$
\mathcal{D}_{h}-\mathcal{D}_{1, h}=-\frac{\epsilon}{2} \sum_{i=1}^{N-1} \int_{0}^{T}\left[\rho_{i+1}^{2}(t)-\rho_{i}^{2}(t)\right]\left[\frac{\partial \varphi}{\partial x}\left(t, x_{i+1 / 2}\right)-\mathrm{d} \varphi_{i+1 / 2}(t)\right] \mathrm{d} t
$$

and observing that

$$
\left|\frac{\partial \varphi}{\partial x}\left(t, x_{i+1 / 2}\right)-\mathrm{d} \varphi_{i+1 / 2}(t)\right| \leq\left\|\frac{\partial^{2} \varphi}{\partial x^{2}}\right\|_{L^{\infty}} h,
$$

we obtain, in conjunction with the Cauchy-Schwarz inequality and the a priori bounds established in Corollary 3.3, and Lemma 3.4, that

$$
\begin{aligned}
\left|\mathcal{D}_{h}-\mathcal{D}_{1, h}\right| & \leq \frac{\epsilon}{2}\left\|\frac{\partial^{2} \varphi}{\partial x^{2}}\right\|_{L^{\infty}}\left(\sum_{i=1}^{N-1} \int_{0}^{T} \Delta x_{i+1 / 2}\left|\mathrm{~d} \rho_{i+1 / 2}\right|^{2} \mathrm{~d} t\right)^{1 / 2} \frac{2\left\|\rho_{h}\right\|_{L^{2}\left(Q_{T}\right)}}{\xi^{1 / 2}} h \\
& \leq C h
\end{aligned}
$$

in the virtue of the estimate Eq. (19).

\section{The cross-diffusion part}

Let us now treat the cross-diffusion part. This term is more complicated since it involves the piecewise constant functions $\rho_{h}$ and $\mathrm{d} U_{h}$, which are not defined on the same mesh. Thus, on the one hand we reformulate the discrete cross-diffusion term $\mathcal{C}_{1, h}$ as $\mathcal{C}_{1, h}=\mathcal{C}_{10, h}+\mathcal{C}_{11, h}$ with

$$
\mathcal{C}_{10, h}=v \sum_{i=1}^{N-1} \int_{0}^{T} \Delta x_{i+1 / 2}(\mathrm{~d} U)_{i+1 / 2}^{-}\left[\rho_{i+1}-\rho_{i}\right] \mathrm{d} \varphi_{i+1 / 2}(t) \mathrm{d} t
$$

and

$$
\mathcal{C}_{11, h}=v \sum_{i=1}^{N-1} \int_{0}^{T} \Delta x_{i+1 / 2} \rho_{i} \mathrm{~d} U_{i+1 / 2} \mathrm{~d} \varphi_{i+1 / 2}(t) \mathrm{d} t
$$

where a direct computation and the application of Corollary 3.3 and Lemma 3.4 yield

$$
\begin{aligned}
\left|\mathcal{C}_{10, h}\right| & \leq v\left\|\frac{\partial \varphi}{\partial x}\right\|_{L^{\infty}}\left\|\mathrm{d} U_{h}\right\|_{L^{2}\left(Q_{T}\right)}\left\|\mathrm{d} \rho_{h}\right\|_{L^{2}\left(Q_{T}\right)} h \\
& \leq C h .
\end{aligned}
$$


On the other hand, the term $\mathcal{C}_{h}$ can be rewritten as

$$
\mathcal{C}_{h}=v \int_{0}^{T} \sum_{i=1}^{N-1} \mathrm{~d} U_{i+1 / 2}(t) \int_{x_{i}}^{x_{i+1}} \rho_{h} \frac{\partial \varphi}{\partial x} \mathrm{~d} x \mathrm{~d} t
$$

Since

$$
\begin{aligned}
\int_{x_{i}}^{x_{i+1}} \rho_{h} \frac{\partial \varphi}{\partial x} \mathrm{~d} x= & \rho_{i}\left[\varphi\left(t, x_{i+1 / 2}\right)-\varphi\left(t, x_{i}\right)\right] \\
& +\rho_{i+1}\left[\varphi\left(t, x_{i+1}\right)-\varphi\left(t, x_{i+1 / 2}\right)\right], \\
= & {\left[\rho_{i}-\rho_{i+1}\right]\left[\varphi\left(t, x_{i+1 / 2}\right)-\varphi\left(t, x_{i}\right)\right] } \\
& +\rho_{i+1}\left[\varphi\left(t, x_{i+1}\right)-\varphi\left(t, x_{i}\right)\right],
\end{aligned}
$$

the term $\mathcal{C}_{h}$ can be decomposed as $\mathcal{C}_{h}=\mathcal{C}_{00, h}+\mathcal{C}_{01, h}$ with

$$
\mathcal{C}_{00, h}=-v \int_{0}^{T} \sum_{i=1}^{N-1} \mathrm{~d} U_{i+1 / 2}\left[\rho_{i+1}-\rho_{i}\right]\left[\varphi\left(t, x_{i+1 / 2}\right)-\varphi\left(t, x_{i}\right)\right] \mathrm{d} t
$$

and

$$
\mathcal{C}_{01, h}=v \int_{0}^{T} \sum_{i=1}^{N-1} \mathrm{~d} U_{i+1 / 2} \rho_{i}\left[\varphi\left(t, x_{i+1}\right)-\varphi\left(t, x_{i}\right)\right] \mathrm{d} t
$$

Similarly to (25), the first term $\mathcal{C}_{00, h}$ can be estimated as

$$
\left|\mathcal{C}_{00, h}\right| \leq C h
$$

whereas the second term $\mathcal{C}_{01, h}$ is compared to $\mathcal{C}_{11, h}$

$$
\begin{aligned}
& \left|\mathcal{C}_{01, h}-\mathcal{C}_{11, h}\right| \\
& \quad \leq v \int_{0}^{T} \sum_{i=1}^{N-1} \Delta x_{i+1 / 2}\left|\mathrm{~d} U_{i+1 / 2}\right| \rho_{i}\left|\frac{\varphi\left(t, x_{i+1}\right)-\varphi\left(t, x_{i}\right)}{\Delta x_{i+1 / 2}}-d \varphi_{i+1 / 2}(t)\right| \mathrm{d} t .
\end{aligned}
$$

Using a second order Taylor expansion of $\varphi$ at $x_{i}$ and $x_{i+1}$, it yields that

$$
\left|\frac{\varphi\left(t, x_{i+1}\right)-\varphi\left(t, x_{i}\right)}{\Delta x_{i+1 / 2}}-d \varphi_{i+1 / 2}(t)\right| \leq C h,
$$

hence we get from Corollary 3.3 and Lemma 3.4 that

$$
\left|\mathcal{C}_{01, h}-\mathcal{C}_{11, h}\right| \leq C h
$$


Gathering Eqs. (25), (26), and (27), we finally obtain that

$$
\left|\mathcal{C}_{h}-\mathcal{C}_{1, h}\right|=\left|\mathcal{C}_{00, h}+\mathcal{C}_{01, h}-\mathcal{C}_{10, h}-\mathcal{C}_{11, h}\right| \leq C h
$$

\section{The advective part}

The evaluation of $\mathcal{A}_{h}-\mathcal{A}_{1, h}$ is along the same lines of the cross-diffusion terms $\mathcal{C}_{h}-\mathcal{C}_{1, h}$ since the latter is treated as an advective term. Hence, thanks to Lemma 4.2, we get that

$$
\left|\mathcal{A}_{h}-\mathcal{A}_{1, h}\right| \leq C h \text {. }
$$

Finally by definition of $\varepsilon(h)$ and using Eq. (23) together with Eqs. (24), (28), and (29), we obtain

$$
\begin{aligned}
|\varepsilon(h)| & =\left|-\left(\mathcal{A}_{1, h}+\mathcal{C}_{1, h}+\mathcal{D}_{1, h}\right)+\mathcal{A}_{h}+\mathcal{C}_{h}+\mathcal{D}_{h}\right| \\
& \leq\left|\mathcal{A}_{h}-\mathcal{A}_{1, h}\right|+\left|\mathcal{C}_{h}-\mathcal{C}_{1, h}\right|+\left|\mathcal{D}_{h}-\mathcal{D}_{1, h}\right| \\
& \leq C h
\end{aligned}
$$

that is, $\varepsilon(h) \rightarrow 0$, when $h \rightarrow 0$, which proves that $(\rho, \eta)$ is a weak solution to Eq. (1). This proves the second item of Theorem 2.4.

Finally the last item concerning the existence of solutions to (1) is a direct consequence of the convergence.

\section{A fully discrete implicit scheme}

In this section we shall comment on a discrete-in-time version of the semi-discrete scheme (9). To this end we replace the time derivative in Eq. (9a) by simple forward differences and obtain the following implicit and fully-discrete scheme

$$
\begin{aligned}
\frac{\rho_{i}^{n+1}-\rho_{i}^{n}}{\Delta t} & =-\frac{\mathcal{F}_{i+1 / 2}^{n+1}-\mathcal{F}_{i-1 / 2}^{n+1}}{\Delta x_{i}} \\
\frac{\eta_{i}^{n+1}-\eta_{i}^{n}}{\Delta t} & =-\frac{\mathcal{G}_{i+1 / 2}^{n+1}-\mathcal{G}_{i-1 / 2}^{n+1}}{\Delta x_{i}}
\end{aligned}
$$

where $\Delta t>0$. System (30) gives rise to two approximating sequences $\left(\rho_{i}^{n}\right)_{1 \leq i \leq N}$ and $\left(\eta_{i}^{n}\right)_{1 \leq i \leq N}$, for $0 \leq n \leq M$ where $M:=\lceil T / \Delta t\rceil$ and the discrete time instances $t^{n}:=n \Delta t$; cf. Theorem 5.1. Here the numerical fluxes are given by

$$
\begin{aligned}
\mathcal{F}_{i+1 / 2}^{n+1}= & {\left[v\left(\mathrm{~d} U^{n+1}\right)_{i+1 / 2}^{+}+\left(\mathrm{d} V_{1}^{n}\right)_{i+1 / 2}^{+}\right] \rho_{i}^{n+1} } \\
& +\left[v\left(\mathrm{~d} U^{n+1}\right)_{i+1 / 2}^{-}+\left(\mathrm{d} V_{1}^{n}\right)_{i+1 / 2}^{-}\right] \rho_{i+1}^{n+1}
\end{aligned}
$$




$$
\begin{aligned}
& -\frac{\epsilon}{2} \frac{\left(\rho_{i+1}^{n+1}\right)^{2}-\left(\rho_{i}^{n+1}\right)^{2}}{\Delta x_{i+1 / 2}} \\
\mathcal{G}_{i+1 / 2}^{n+1}= & {\left[v\left(\mathrm{~d} U^{n+1}\right)_{i+1 / 2}^{+}+\left(\mathrm{d} V_{2}^{n}\right)_{i+1 / 2}^{+}\right] \eta_{i}^{n+1} } \\
& +\left[v\left(\mathrm{~d} U^{n+1}\right)_{i+1 / 2}^{-}+\left(\mathrm{d} V_{2}^{n}\right)_{i+1 / 2}^{-}\right] \eta_{i+1}^{n+1} \\
& -\frac{\epsilon}{2} \frac{\left(\eta_{i+1}^{n+1}\right)^{2}-\left(\eta_{i}^{n+1}\right)^{2}}{\Delta x_{i+1 / 2}}
\end{aligned}
$$

for $i=1, \ldots, N-1$, with the numerical no-flux boundary condition

$$
\mathcal{F}_{1 / 2}^{n+1}=\mathcal{F}_{N+1 / 2}^{n+1}=0, \quad \text { and } \mathcal{G}_{1 / 2}^{n+1}=\mathcal{G}_{N+1 / 2}^{n+1}=0,
$$

for $n=0, \ldots, M$. Recall that

$$
\left(\mathrm{d} U^{n+1}\right)_{i+1 / 2}^{ \pm}=\left(\frac{\left(\rho_{i+1}^{n+1}+\eta_{i+1}^{n+1}\right)-\left(\rho_{i}^{n+1}+\eta_{i}^{n+1}\right)}{\Delta x_{i+1 / 2}}\right)^{ \pm},
$$

and

$$
\begin{aligned}
\left(\mathrm{d} V_{k}^{n}\right)_{i+1 / 2}^{ \pm}= & \left(-\sum_{j=1}^{N} \int_{C_{j}} \frac{W_{k 1}\left(x_{i+1}-y\right)-W_{k 1}\left(x_{i}-y\right)}{\Delta x_{i+1 / 2}} \rho_{j}^{n} \mathrm{~d} y\right. \\
& \left.-\sum_{j=1}^{N} \int_{C_{j}} \frac{W_{k 2}\left(x_{i+1}-y\right)-W_{k 2}\left(x_{i}-y\right)}{\Delta x_{i+1 / 2}} \eta_{j}^{n} \mathrm{~d} y\right)^{ \pm}
\end{aligned}
$$

for $k=1,2$.

Similarly to Definition 2.3 we define the piecewise constant interpolation by

$$
\rho_{h}(t, x):=\rho_{i}^{n}, \quad \text { and } \quad \eta_{h}(t, x):=\eta_{i}^{n},
$$

for all $(t, x) \in\left[t^{n}, t^{n+1}\right) \times C_{i}$, with $i=1, \ldots, N$, and $n=0, \ldots, M$. Moreover, we define the discrete approximation of the spatial gradients as

$$
\mathrm{d}_{x} \rho_{h}(t, x)=\frac{\rho_{i+1}^{n}-\rho_{i}^{n}}{\Delta x_{i+1 / 2}}, \quad \text { and } \quad \mathrm{d}_{x} \eta_{h}(t, x)=\frac{\eta_{i+1}^{n}-\eta_{i}^{n}}{\Delta x_{i+1 / 2}},
$$

for $(t, x) \in\left[t^{n}, t^{n+1}\right) \times\left[x_{i}, x_{i+1}\right)$, for $i=1, \ldots, N-1$ and $n=0, \ldots, M$. As above, we set the discrete gradients to zero on $\left(a, x_{1}\right)$ and $\left(x_{N}, b\right)$. Furthermore, we define the discrete time derivative as

$$
\mathrm{d}_{t} \rho_{h}(t, x)=\frac{\rho_{i}^{n+1}-\rho_{i}^{n}}{\Delta t}, \quad \text { and } \quad \mathrm{d}_{t} \eta_{h}(t, x)=\frac{\eta_{i}^{n}-\eta_{i}^{n}}{\Delta t},
$$

for $(t, x) \in\left[t^{n}, t^{n+1}\right) \times C_{i}$, for $i=1, \ldots, N$ and $n=0, \ldots, M-1$. 
Theorem 5.1 (Existence and uniqueness result) Let $\rho_{i}^{0}, \eta_{i}^{0}$ be nonnegative initial data with mass $m_{1}$ and $m_{2}$, respectively, and assume the following time step restriction condition

$$
16\left(m_{1}+m_{2}\right) \frac{\Delta t}{(\xi h)^{3}}<1 .
$$

Then there exists a unique nonnegative solution $\left(\rho_{i}^{n}, \eta_{i}^{n}\right)$ to scheme (30), (30a) and (30b).

Proof We show existence first and prove uniqueness later. Suppose we are given $\left(\rho_{i}^{n}\right)_{1 \leq i \leq N}$ and $\left(\eta_{i}^{n}\right)_{1 \leq i \leq N}$ from some previous iteration. In order to construct the next iteration we shall employ Brouwer's fixed point theorem. It is easy to verify that the set

$$
\begin{gathered}
\mathcal{X}:=\left\{(\rho, \eta) \in \mathbb{R}^{2 N} \mid \forall 1 \leq i \leq N: \rho_{i}, \eta_{i} \geq 0,\right. \\
\left.\sum_{i=1}^{N} \Delta x_{i} \rho_{i} \leq m_{1}, \text { and } \sum_{i=1}^{N} \Delta x_{i} \eta_{i} \leq m_{2}\right\},
\end{gathered}
$$

is a convex and compact subset of $\mathbb{R}^{N} \times \mathbb{R}^{N}$. Hence, we define the fixed point operator $\mathfrak{S}: \mathcal{X} \rightarrow \mathbb{R}^{N} \times \mathbb{R}^{N}$ by setting $\left(\rho^{\star}, \eta^{\star}\right)=\mathfrak{S}(\rho, \eta)$ where $\left(\rho^{\star}, \eta^{\star}\right)$ are implicitly given as

$$
\rho_{i}^{\star}=\rho_{i}^{n}-\frac{\Delta t}{\Delta x_{i}}\left(\mathcal{F}_{i+1 / 2}^{\star}-\mathcal{F}_{i-1 / 2}^{\star}\right), \quad \eta_{i}^{\star}=\eta_{i}^{n}-\frac{\Delta t}{\Delta x_{i}}\left(\mathcal{G}_{i+1 / 2}^{\star}-\mathcal{G}_{i-1 / 2}^{\star}\right)
$$

for any $(\rho, \eta) \in \mathcal{X}$, where $\mathcal{F}^{\star}$ and $\mathcal{G}^{\star}$ denote the numerical fluxes

$$
\begin{aligned}
\mathcal{F}_{i+1 / 2}^{\star}= & {\left[v(\mathrm{~d} U)_{i+1 / 2}^{+}+\left(\mathrm{d} V_{1}^{n}\right)_{i+1 / 2}^{+}\right] \rho_{i}^{\star} } \\
& +\left[v(\mathrm{~d} U)_{i+1 / 2}^{-}+\left(\mathrm{d} V_{1}^{n}\right)_{i+1 / 2}^{-}\right] \rho_{i+1}^{\star} \\
& -\frac{\epsilon\left(\rho_{i+1}+\rho_{i}\right)}{2} \frac{\rho_{i+1}^{\star}-\rho_{i}^{\star}}{\Delta x_{i+1 / 2}}, \\
\mathcal{G}_{i+1 / 2}^{\star}= & {\left[v(\mathrm{~d} U)_{i+1 / 2}^{+}+\left(\mathrm{d} V_{2}^{n}\right)_{i+1 / 2}^{+}\right] \eta_{i}^{\star} } \\
& +\left[v(\mathrm{~d} U)_{i+1 / 2}^{-}+\left(\mathrm{d} V_{2}^{n}\right)_{i+1 / 2}^{-}\right] \eta_{i+1}^{\star} \\
& -\frac{\epsilon\left(\eta_{i+1}+\eta_{i}\right)}{2} \frac{\eta_{i+1}^{\star}-\eta_{i}^{\star}}{\Delta x_{i+1 / 2}},
\end{aligned}
$$

for $i=1, \ldots, N-1$ where $\mathrm{d} U$ is computed from $(\rho, \eta)$, with the numerical no-flux boundary condition

$$
\mathcal{F}_{1 / 2}^{\star}=\mathcal{F}_{N+1 / 2}^{\star}=0, \quad \text { and } \mathcal{G}_{1 / 2}^{\star}=\mathcal{G}_{N+1 / 2}^{\star}=0 \text {. }
$$


Notice that for any given $(\rho, \eta) \in \mathcal{X}$, the viscosity terms in front of the discrete gradients involved in the definition of the fluxes $\mathcal{F}_{i+1 / 2}^{\star}$ and $\mathcal{G}_{i+1 / 2}^{\star}$ are indeed nonnegative, hence the couple $\left(\rho^{\star}, \eta^{\star}\right)$ is well defined since it corresponds to the unique solution of a classical fully implicit scheme in time with an upwind discretisation for the convective terms and a centred approximation for diffusive terms [29]. Moreover, since $\rho^{n}$ and $\eta^{n}$ are nonnegative and using the monotonicity of the numerical flux with respect to $\left(\rho^{\star}, \eta^{\star}\right)$, we prove that both densities $\rho^{\star}$ and $\eta^{\star}$ are also nonnegative. Furthermore, using the nonnegativity and the no-flux conditions, we get

$$
\left\|\rho^{\star}\right\|_{L^{1}}=\sum_{i=1}^{N} \Delta x_{i} \rho_{i}^{\star}=m_{1}, \quad \text { and }\left\|\eta^{\star}\right\|_{L^{1}}=\sum_{i=1}^{N} \Delta x_{i} \eta_{i}^{\star}=m_{2}
$$

which yields that $\mathfrak{S}(\mathcal{X}) \subset \mathcal{X}$. Finally, $\mathfrak{S}$ is continuous as the composition of continuous functions. Thus, we may apply Brouwer's fixed point theorem to infer the existence of a fixed point, $\left(\rho^{n+1}, \eta^{n+1}\right)$. It now remains to show uniqueness of the fixed point.

To treat in a systematic way the boundary conditions and simplify the presentation, we define ghost values for $(\rho, \eta)$ by setting for $\alpha \in\{\rho, \eta\}$, and $k \in\{1,2\}$,

$$
\alpha_{N+1}=\alpha_{N} \quad \alpha_{0}=\alpha_{1} \quad \text { and } \quad\left(\mathrm{d} V_{k}^{n}\right)_{N+1 / 2}=\left(\mathrm{d} V_{k}^{n}\right)_{1 / 2}=0
$$

Then we consider two solutions $(\tilde{\rho}, \tilde{\eta})$ and $(\rho, \eta)$ to $(30)$. Setting $h(x):=x^{2} / 2$, $s:=\rho-\tilde{\rho}$ and $r:=\eta-\tilde{\eta}$, we get after substituting the two solutions to (30), for $i=1, \ldots, N$,

$$
\begin{aligned}
s_{i}= & -\frac{\Delta t}{\Delta x_{i}}\left(\left(\mathrm{~d} V_{1}^{n}\right)_{i+1 / 2}^{+} s_{i}+\left(\mathrm{d} V_{1}^{n}\right)_{i+1 / 2}^{-} s_{i+1}-\left(\mathrm{d} V_{1}^{n}\right)_{i-1 / 2}^{+} s_{i-1}-\left(\mathrm{d} V_{1}^{n}\right)_{i-1 / 2}^{-} s_{i}\right) \\
& +\frac{\Delta t}{\Delta x_{i}}\left(\frac{\left[h\left(\rho_{i+1}\right)-h\left(\tilde{\rho}_{i+1}\right)\right]-\left[h\left(\rho_{i}\right)-h\left(\tilde{\rho}_{i}\right)\right]}{\Delta x_{i+1 / 2}}\right. \\
& \left.-\frac{\left[h\left(\rho_{i}\right)-h\left(\tilde{\rho}_{i}\right)\right]-\left[h\left(\rho_{i-1}\right)-h\left(\tilde{\rho}_{i-1}\right)\right]}{\Delta x_{i-1 / 2}}\right) \\
& -\frac{\Delta t}{\Delta x_{i}}\left((\mathrm{~d} U)_{i+1 / 2}^{+} s_{i}+(\mathrm{d} U)_{i+1 / 2}^{-} s_{i+1}-(\mathrm{d} U)_{i-1 / 2}^{+} s_{i-1}-(\mathrm{d} U)_{i-1 / 2}^{-} s_{i}\right) \\
& -\frac{\Delta t}{\Delta x_{i}}\left(\left[(\mathrm{~d} U)_{i+1 / 2}^{+}-(\mathrm{d} \tilde{U})_{i+1 / 2}^{+}\right] \tilde{\rho}_{i}+\left[(\mathrm{d} U)_{i+1 / 2}^{-}-(\mathrm{d} \tilde{U})_{i+1 / 2}^{-}\right] \tilde{\rho}_{i+1}\right) \\
& +\frac{\Delta t}{\Delta x_{i}}\left(\left[(\mathrm{~d} U)_{i-1 / 2}^{+}-(\mathrm{d} \tilde{U})_{i-1 / 2}^{+}\right] \tilde{\rho}_{i-1}+\left[(\mathrm{d} U)_{i-1 / 2}^{-}-(\mathrm{d} \tilde{U})_{i-1 / 2}^{-}\right] \tilde{\rho}_{i}\right)
\end{aligned}
$$

and a similar relation for $\left(r_{i}\right)_{1 \leq i \leq N}$. Applying a Taylor expansion on $h(\rho)=h(\tilde{\rho})+$ $h^{\prime}(\hat{\rho}) s$, with $\hat{\rho}$ a convex combination of $\rho$ and $\tilde{\rho}$, we may write

$$
\begin{aligned}
& \left(\Delta x_{i}+\Delta t A_{i}\right) s_{i} \\
& \quad=\Delta t\left(B_{i-1} s_{i-1}+C_{i+1} s_{i+1}\right)
\end{aligned}
$$




$$
\begin{aligned}
& -\Delta t\left(\left[(\mathrm{~d} U)_{i+1 / 2}^{+}-(\mathrm{d} \tilde{U})_{i+1 / 2}^{+}\right] \tilde{\rho}_{i}+\left[(\mathrm{d} U)_{i+1 / 2}^{-}-(\mathrm{d} \tilde{U})_{i+1 / 2}^{-}\right] \tilde{\rho}_{i+1}\right) \\
& +\Delta t\left(\left[(\mathrm{~d} U)_{i-1 / 2}^{+}-(\mathrm{d} \tilde{U})_{i-1 / 2}^{+}\right] \tilde{\rho}_{i-1}+\left[(\mathrm{d} U)_{i-1 / 2}^{-}-(\mathrm{d} \tilde{U})_{i-1 / 2}^{-}\right] \tilde{\rho}_{i}\right),
\end{aligned}
$$

where $A_{i}, B_{i-1}, C_{i+1}$ are nonnegative coefficients given by

$$
\begin{aligned}
A_{i}= & +\left(\mathrm{d} V_{1}^{n}\right)_{i+1 / 2}^{+}-\left(\mathrm{d} V_{1}^{n}\right)_{i-1 / 2}^{-}+(\mathrm{d} U)_{i+1 / 2}^{+}-(\mathrm{d} U)_{i-1 / 2}^{-} \\
& +\frac{h^{\prime}\left(\hat{\rho}_{i}\right)}{\Delta x_{i+1 / 2}}+\frac{h^{\prime}\left(\hat{\rho}_{i}\right)}{\Delta x_{i-1 / 2}}, \\
B_{i-1}= & +\left(\mathrm{d} V_{1}^{n}\right)_{i-1 / 2}^{+}+(\mathrm{d} U)_{i-1 / 2}^{+}+\frac{h^{\prime}\left(\hat{\rho}_{i-1}\right)}{\Delta x_{i-1 / 2}}, \\
C_{i+1}= & -\left(\mathrm{d} V_{1}^{n}\right)_{i+1 / 2}^{-}-(\mathrm{d} U)_{i+1 / 2}^{-}+\frac{h^{\prime}\left(\hat{\rho}_{i+1}\right)}{\Delta x_{i+1 / 2}} .
\end{aligned}
$$

Now, we multiply equation (34) by $\operatorname{sign}\left(s_{i}\right)$ and sum over $i=1, \ldots, N$, hence using that $x \mapsto x^{ \pm}$is Lipschitz continuous and observing that $A_{i}=B_{i}+C_{i}$, with $A_{i}, B_{i}, C_{i} \geq 0$, it yields

$$
\begin{aligned}
\sum_{i=1}^{N} \Delta x_{i}\left|s_{i}\right| \leq & 2 \Delta t\|\tilde{\rho}\|_{\infty} \sum_{i=1}^{N}\left(\frac{\left|s_{i+1}\right|+\left|r_{i+1}\right|+\left|s_{i}\right|+\left|r_{i}\right|}{\Delta x_{i+1 / 2}}\right. \\
& \left.+\frac{\left|s_{i-1}\right|+\left|r_{i-1}\right|+\left|s_{i}\right|+\left|r_{i}\right|}{\Delta x_{i-1 / 2}}\right)
\end{aligned}
$$

and in a similar way,

$$
\begin{aligned}
\sum_{i=1}^{N} \Delta x_{i}\left|r_{i}\right| \leq & 2 \Delta t\|\tilde{\eta}\|_{\infty} \sum_{i=1}^{N}\left(\frac{\left|r_{i+1}\right|+\left|s_{i+1}\right|+\left|r_{i}\right|+\left|s_{i}\right|}{\Delta x_{i+1 / 2}}\right. \\
& \left.+\frac{\left|r_{i-1}\right|+\left|s_{i-1}\right|+\left|r_{i}\right|+\left|s_{i}\right|}{\Delta x_{i-1 / 2}}\right) .
\end{aligned}
$$

Gathering these latter inequalities and from (4), it gives that

$$
\sum_{i=1}^{N} \Delta x_{i}\left(\left|s_{i}\right|+\left|r_{i}\right|\right) \leq 16\|(\tilde{\rho}, \tilde{\eta})\|_{\infty} \frac{\Delta t}{(\xi h)^{2}} \sum_{i=1}^{N} \Delta x_{i}\left(\left|s_{i}\right|+\left|r_{i}\right|\right) .
$$

Finally, from the nonnegativity and the preservation of mass, we have

$$
\|(\tilde{\rho}, \tilde{\eta})\|_{\infty} \leq \frac{m_{1}+m_{2}}{\xi h},
$$

hence under the condition (31), we conclude that $s=r=0$ and the uniqueness follows. 
It is worth to mention here that the condition (31) is not optimal since we only use the discrete $L^{1}$-estimate on $\rho$ and $\eta$ to control the discrete gradient and the $L^{\infty}$-norm.

We are now in position to state for (30), (30a) and (30b) an analogous results to the semi-discrete case

Theorem 5.2 (Convergence to a weak solution of the implicit Euler discretisation) Under the assumptions of Theorem 5.1, let $\rho_{0}, \eta_{0} \in L_{+}^{1}(a, b) \cap L_{+}^{\infty}(a, b)$ be some initial data and $Q_{T}:=(0, T) \times(a, b)$ as above. Then, given two nonnegative sequences $\left(\rho_{i}^{n}\right)_{1 \leq i \leq N}$ and $\left(\eta_{i}^{n}\right)_{1 \leq i \leq N}$ satisfying (30), (30a) and (30b), for any $n \in\{0, \ldots, M\}$, then

(i) up to a subsequence, the piecewise constant approximations converge strongly in $L^{2}\left(Q_{T}\right)$ to $(\rho, \eta) \in L^{2}\left(Q_{T}\right)$, where $(\rho, \eta)$ is a weak solution as in Definition 2.1. Furthermore we have $\rho, \eta \in L^{2}\left(0, T ; H^{1}(a, b)\right)$;

(ii) in particular, system (1) has a weak solution.

Sketch of the proof of Theorem 5.2 It is easily observed that the total mass is conserved due to the discrete no-flux boundary conditions, cf. (30b). Together with the nonnegativity we were able to prove a semi-discrete version of the energy estimate Eq. (2) which is at the heart of the convergence result. Similarly as above, we are able to prove a fully discrete version of the energy estimate which then reads

$$
\begin{aligned}
& \sum_{n=0}^{M} \Delta t \sum_{i=1}^{N} \Delta x_{i} \frac{\rho_{i}^{n+1} \log \rho_{i}^{n+1}-\rho_{i}^{n} \log \rho_{i}^{n}}{\Delta t}+\frac{\eta_{i}^{n+1} \log \eta_{i}^{n+1}-\eta_{i}^{n} \log \eta_{i}^{n}}{\Delta t} \\
& \quad+\sum_{n=0}^{M} \Delta t \sum_{i=1}^{N-1} \Delta x_{i+1 / 2}\left[v\left|\mathrm{~d} U_{i+1 / 2}^{n+1}\right|^{2}+\frac{\epsilon}{4}\left(\left|\mathrm{~d} \rho_{i+1 / 2}^{n+1}\right|^{2}+\left|\mathrm{d} \eta_{i+1 / 2}^{n+1}\right|^{2}\right)\right] \leq C,
\end{aligned}
$$

with $C>0$ as in Corollary 3.3. The inequality follows from the convexity of $x(\log x-$ 1) since

$$
\rho_{i}^{n+1}\left(\log \rho_{i}^{n+1}-1\right)-\rho_{i}^{n}\left(\log \rho_{i}^{n}-1\right) \leq \log \left(\rho_{i}^{n+1}\right)\left(\rho_{i}^{n+1}-\rho_{i}^{n}\right) .
$$

Multiplying this expression by $\Delta x_{i} / \Delta t$ and summing over $i=1, \ldots N$ and $n=$ $0, \ldots, M$ we obtain

$$
\begin{aligned}
& \sum_{n=0}^{M} \Delta t \sum_{i=1}^{N} \Delta x_{i} \frac{\rho_{i}^{n+1}\left(\log \rho_{i}^{n+1}-1\right)-\rho_{i}^{n}\left(\log \rho_{i}^{n}-1\right)}{\Delta t} \\
& \quad \leq \sum_{n=0}^{M} \Delta t \sum_{i=1}^{N} \Delta x_{i} \log \left(\rho_{i}^{n+1}\right) \frac{\rho_{i}^{n+1}-\rho_{i}^{n}}{\Delta t} .
\end{aligned}
$$

The right-hand side is then substituted by the scheme and simplified along the lines of the proof of Lemma 3.2. Since the computations are exactly the same we omit them 
here for brevity and only note that it is important to set

$$
\tilde{\rho}_{i+1 / 2}^{n+1}:= \begin{cases}\frac{\rho_{i+1}^{n+1}-\rho_{i}^{n+1}}{\log \rho_{i+1}^{n+1}-\log \rho_{i}^{n+1}}, & \text { if } \rho_{i}^{n+1} \neq \rho_{i+1}^{n+1}, \\ \frac{\rho_{i}^{n+1}+\rho_{i+1}^{n+1}}{2}, & \text { else, }\end{cases}
$$

to obtain the right sign in the numerical artefacts in Eq. (14), which now read

$$
\begin{aligned}
& \left(\rho_{i}^{n+1}-\tilde{\rho}_{i+1 / 2}^{n+1}\right) \mathrm{d} \log \rho_{i+1 / 2}^{n+1}\left(v(\mathrm{~d} U n+1)_{i+1 / 2}^{+}+\left(\mathrm{d} V_{1}^{n}\right)_{i+1 / 2}^{+}\right) \leq 0, \\
& \left(\rho_{i+1}^{n+1}-\tilde{\rho}_{i+1 / 2}^{n+1}\right) \mathrm{d} \log \rho_{i+1 / 2}^{n+1}\left(v\left(\mathrm{~d} U^{n+1}\right)_{i+1 / 2}^{-}+\left(\mathrm{d} V_{1}^{n}\right)_{i+1 / 2}^{-}\right) \leq 0 .
\end{aligned}
$$

Following the lines of the proof Lemma 3.2 and Corollary 3.3 we obtain the fully discrete a priori bounds

$$
\sum_{n=0}^{N} \Delta t \sum_{i=1}^{N-1} \Delta x_{i+1 / 2}\left(\left|\mathrm{~d}_{x} \rho_{i+1 / 2}^{n+1}\right|^{2}+\left|\mathrm{d}_{x} \eta_{i+1 / 2}^{n+1}\right|^{2}+\left|\mathrm{d}_{x} U_{i+1 / 2}^{n+1}\right|^{2}\right) \leq C
$$

for some constant $C>0$, where we used ' $\mathrm{d}_{x}$ ' to denote the discrete spatial gradient as before. Again, an application of Aubin-Lions Lemma provides relative compactness in the space $L^{2}((0, T) \times(a, b))$ of the piecewise constant interpolations $\rho_{h}, \eta_{h}$. As above, the discrete gradients are uniformly bounded in $L^{2}((0, T) \times(a, b))$ and their weak convergence is a consequence of the Banach-Alaoglu Theorem. Identifying the limits as a weak solution to system (1) is shown in the same way as above and we leave it as an exercise for the reader.

Remark 1 (Explicit scheme) We do not consider an explicit scheme here since its analysis is much more complicated due to the lack of uniform estimates. Indeed, an explicit scheme requires a CFL condition on the time step which appears in the stability analysis or the energy estimate provided in Lemma 3.2. In our case, it leads to

$$
\frac{\rho^{n+1} \log \rho^{n+1}-\rho^{n} \log \rho^{n}}{\Delta t}=\frac{\rho^{n+1}-\rho^{n}}{\Delta t}\left(\log \rho^{n}-1\right)+\frac{\left(\rho^{n+1}-\rho^{n}\right)^{2}}{\left|\rho^{n+1 / 2}\right|^{2} \Delta t},
$$

where $\rho^{n+1 / 2}$ belongs to the interval $\left[\rho^{n}, \rho^{n+1}\right]$ or $\left[\rho^{n+1}, \rho^{n}\right]$. The control of this reminder term would require some lower bounds estimates on the density $\rho$ (see for instance [30]).

\section{Numerical examples and validation}

In this section we perform some numerical simulations of system (1) using our scheme, Eqs. (9). In Sect. 6.1 we test our scheme by computing the error between the numerical simulation and a benchmark solution on a finer grid. Furthermore we determine the 
numerical convergence order. In Sect. 6.2 we compute the numerical stationary states of system (1) and discuss the implication of different cross-diffusivities and the selfdiffusivities, respectively.

Let us note here, that in the case of no regularising porous-medium diffusion, i.e. $\epsilon=0$, and certain singular potentials the stationary states of system (1) are even known explicitly [19]. This allows us to compare the numerical solution directly to the analytical stationary state in Sect. 6.2.1.

Throughout the remainder of this section we apply the scheme (9) to system (1) using different self-diffusions, $\epsilon$, and cross-diffusions, $v$.

\subsection{Error and numerical order of convergence}

This section is dedicated to validating our main convergence result, Theorem 2.4. Due to the lack of explicit solutions we compute the numerical solution on a fine grid and consider it a benchmark solution. We then compute numerical approximations on coarser grids and study the error in order to obtain the numerical convergence order.

In all our simulations we use a fourth order Runge-Kutta scheme to solve the ordinary differential equations Eq. (9) with initial data Eq. (5). The discrepancy between the benchmark solution and numerical solutions on coarser grids is measured by the error

$$
e:=\left(\Delta t \sum_{k=1}^{M}\left(\Delta x \sum_{i=1}^{N}\left|\rho_{\mathrm{ex}}\left(t_{k}, x_{i}\right)-\rho\left(t_{k}, x_{i}\right)\right|^{2}+\left|\eta_{\mathrm{ex}}\left(t_{k}, x_{i}\right)-\eta\left(t_{k}, x_{i}\right)\right|^{2}\right)\right)^{1 / 2}
$$

Here $\rho_{\mathrm{ex}}, \eta_{\mathrm{ex}}$ denote the benchmark solutions. We use this quantity to study the convergence of our scheme as the grid size decreases.

\subsubsection{No non-local interactions}

Let us begin with the purely diffusive system. We consider system (1) without any interactions, i.e. $W_{i j} \equiv 0$, for $i, j=1,2$, and we choose $v=0.5$ and $\epsilon=0.1$.

In Fig. 2 we present the convergence result as we decrease the grid size. We computed a benchmark solution on a grid of $\Delta x=2^{-10}$ on the time interval $[0,10]$ with $\Delta t=0.05$. Figure $2 \mathrm{a}$ shows the convergence for symmetric initial data whereas Fig. $2 \mathrm{~b}$ shows the convergence of the same system in case of asymmetric initial data. In both cases we overlay a line of slope one and we conclude that the numerical convergence is of order one.

\subsubsection{Gaussian cross-interactions}

Next, we add non-local self-interaction and cross-interactions. We choose smooth Gaussians with different variances. These potentials, like the related, more singular Morse potentials, are classical in mathematical biology since oftentimes the availability of sensory information such as sight, smell or hearing is spatially limited [18,20,27]. 


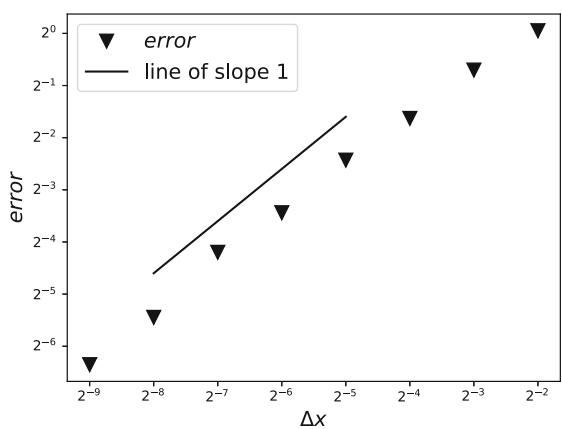

(a) Symmetric initial data. $\rho_{0}(x)$ $=\eta_{0}(x)=\mathbb{1}_{[7,10]}$.

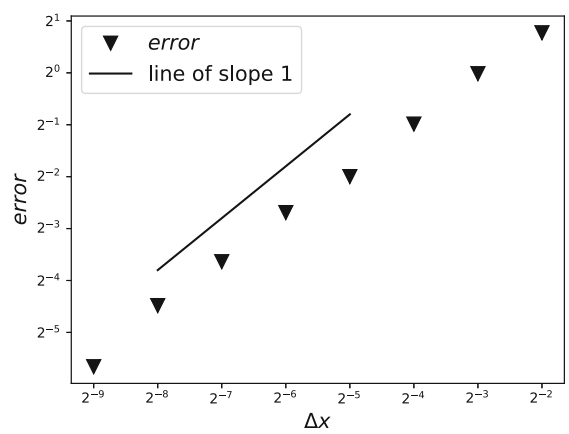

(b) Asymmetric initial data. $\rho_{0}(x)$ $=\mathbb{1}_{[5,7]}$ and $\eta_{0}(x)=\mathbb{1}_{[10,12]}$.

Fig. 2 In the purely diffusive system all interaction kernels are set to zero. Both graphs show the convergence to the benchmark solution. The triangular markers denote the discrepancy between the numerical solution and the benchmark solution. A line of slope one is superimposed for the ease of comparison. On the left we start the system with symmetric initial conditions, on the right we start with asymmetric initial data. In both cases the scheme has numerical convergence order 1

For the intraspecific interaction we use

$$
W_{11}(x)=W_{22}(x)=1-\exp \left(-\frac{|x|^{4}}{4 \times 0.1}\right)
$$

while we choose

$$
W_{12}(x)=-W_{21}(x)=1-\exp \left(-\frac{|x|^{2}}{2 \times 0.1}\right),
$$

for the interspecific interactions.

We consider system (1) with the diffusive coefficients $v=0.4$ and $\epsilon \in\{0.1,0.5\}$, and we initialise the system with

$$
\rho(x)=\eta(x)=c((s-6.5)(9.5-s))^{+},
$$

on the domain $[0,9]$. Here the constant $c$ is such that $\rho$ and $\eta$ have unit mass. Figure 3 depicts the simulation with Gaussian kernels as interaction potentials. In Fig. 3a, b we present the error plots corresponding to $\epsilon \in\{0.1,0.5\}$. Again we observe convergence to the reference solution with a first order accuracy in space.

\subsection{General behaviour of solutions and stationary states}

In this section we aim to study the asymptotic behaviour of system (1) numerically. Let us begin by going back to the set up of Sect.6.1.2. We note that the potentials were chosen in such a way that there is an attractive intraspecific force, and the crossinteractions are chosen as attractive-repulsive explaining the segregation observed in 


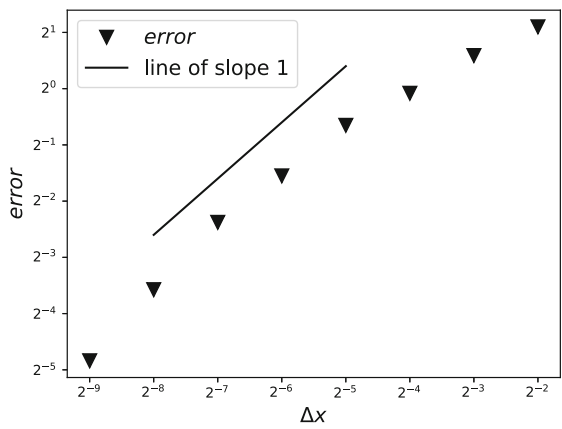

(a) Convergence to benchmark solution for individual diffusion constant $\epsilon=0.1$.

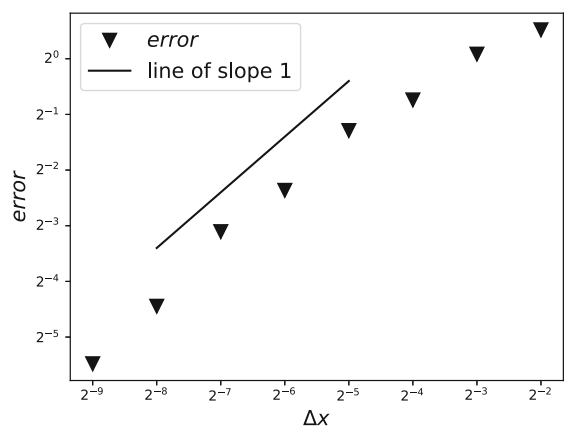

(b) Convergence to benchmark solution for individual diffusion constant $\epsilon=0.5$.

Fig. 3 We choose Gaussian interaction kernels of different strengths and ranges for the self-interaction and the cross-interaction, respectively. The graphs show the numerical convergence order in the cases of $\epsilon=0.1$ (left), and $\epsilon=0.5$ (right), respectively

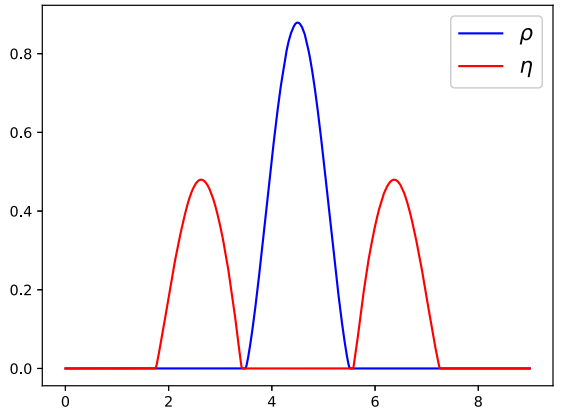

(a) Stationary state $(\epsilon=0.1)$.

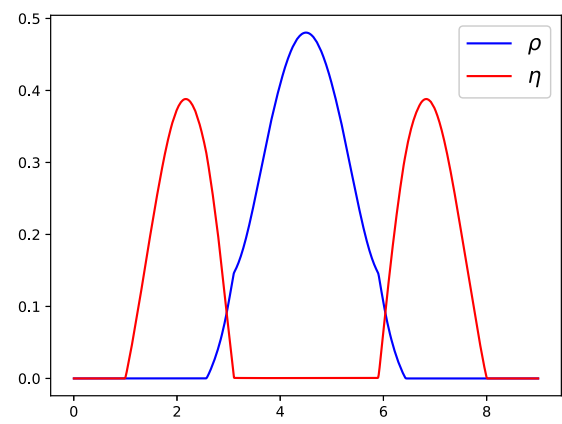

(b) Stationary state $(\epsilon=0.5)$.

Fig. 4 We choose Gaussian interaction kernels of different strengths and ranges for the self-interaction and the cross-interaction, respectively. The graphs correspond to the simulationed stationary states for self-diffusivities $\epsilon=0.1$, and $\epsilon=0.5$, respectively

Fig. 4a. For larger self-diffusivity, $\epsilon=0.5$, we see that some mixing occurs. In the absence of any individual diffusion we would have expected adjacent species with a jump discontinuity at their shared boundary [19]. However, this phenomenon is no longer possible as we have a control on the gradients of each individual species, by Lemma 3.3, rendering jumps impossible thus explaining the continuous transition.

In the subsequent section we shall push our scheme even further by dropping the smoothness assumption on our potentials.

\subsubsection{Case of singular potentials}

In this section we go beyond the limit of what we could prove in this paper. On the one hand we consider more singular potentials and on the other hand we consider vanishing individual diffusion. We study system (1) for $\epsilon \in\{0,0.02,0.04,0.06,0.09\}$, and 


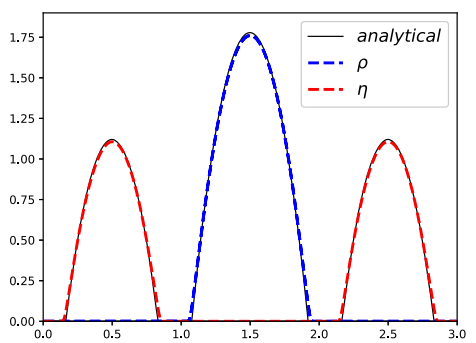

(a) In the case $\nu=0.05, \epsilon=0$, we obtain a great agree-a ment of the numerically computed stationary states and the analytical stationary states described in [19].

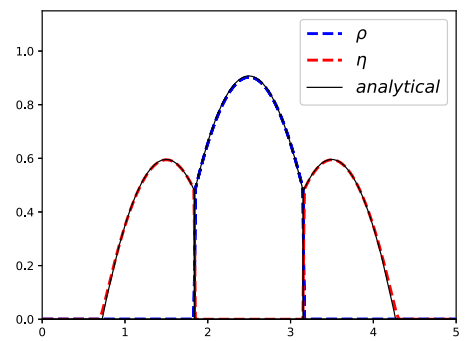

(c) The case $\nu=0.5, \epsilon=0$ leads to adjacent stationary states. Again we see an excellent agreement of the numerical stationary states and the analytical ones [19].

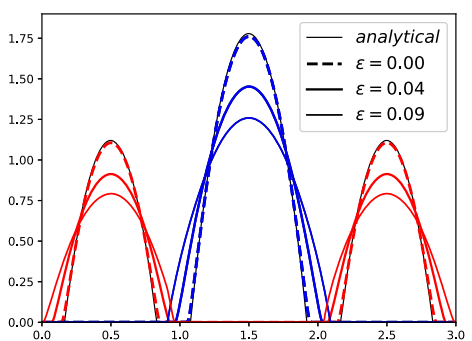

(b) Adding individual diffusion may still lead to segregated stationary states. However both specie remain continuous as they mix.

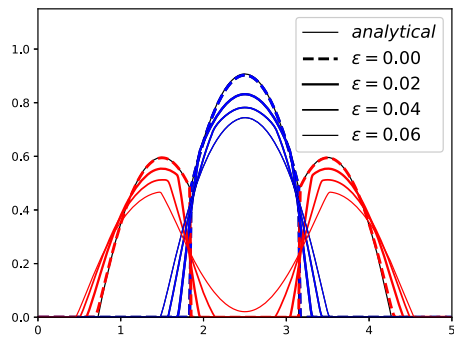

(d) The regularising effect of the individual diffusion, by Corollary 3.3 , becomes apparent immediately. Instantaneously both species become continuous as they start to intermingle in a small region. This region grows as we keep increasing the individual diffusion.

Fig. 5 We pushed our numerical scheme to see how it performs in regimes in which we are unable to prove convergence. We chose Newtonian cross-interactions in the attractive-repulsive case. The red curves denote the symmetric stationary states of $\eta$ while the blue curves are the stationary distributions of $\rho$. The different line widths and styles correspond to varying values of $\epsilon$ (colour figure online)

$v \in\{0.05,0.5\}$. Here the potentials are given by

$$
W_{11}(x)=W_{22}(x)=x^{2} / 2
$$

for the self-interaction terms and

$$
W_{12}(x)=|x|= \pm W_{21}(x)
$$

for the cross-interactions. The system is posed on the domain $[0,5]$ with a grid size of $\Delta x=2^{-8}$. Note that the case of $\epsilon=0$ corresponds to the absence of individual diffusion, see Fig. 5a, c. By virtue of Corollary 3.3, it is the individual diffusion that regularises the stationary states, in the sense that we will not observe any discontinuities in either $\rho$ or $\eta$. As we add individual diffusion we can see the immediate regularisation. While stationary states may still remain segregated, as it is shown in Fig. 5b, adjacent solutions are not possible anymore (see Fig. 5d). 


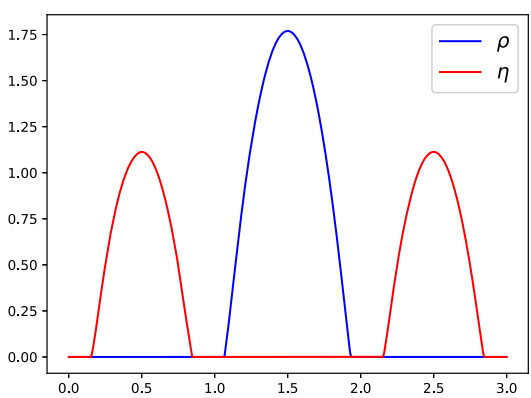

(a) Strictly segregated stationary state in the absence of individual diffusion, $\epsilon=0$, and Newtonian potentials.

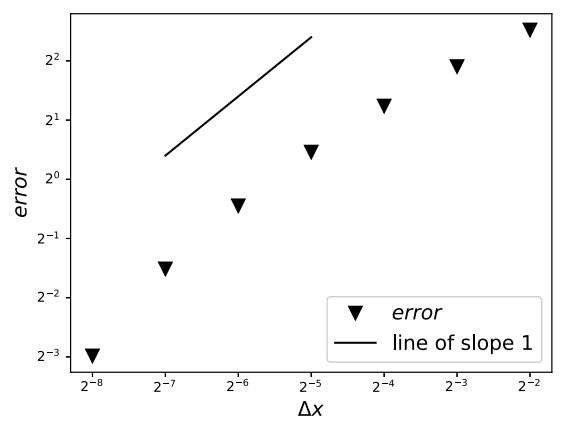

(b) Convergence to benchmark solution in the case of $\epsilon=0$ and Newtonian attractiverepulsive potentials.

Fig. 6 Stationary state and numerical convergence order in the case of Newtonian attractive-repulsive crossinteractions. Even though our estimates fail in the analysis above we observe a numerical convergence order of one

In the case of attractive-repulsive interspecific interactions, i.e.

$$
W_{12}(x)=|x|=-W_{21}(x)
$$

we expect both species to segregate [19]. We initialise the system with the following symmetric initial data

$$
\rho(x)=\eta(x)=c((x-3)(5-x))^{+},
$$

as symmetric initial data are known to approach stationary states [19]. Here the constant $c$ normalises the mass of $\rho$ and $\eta$ to one.

Figure 5a, c show our scheme performs well even in regimes we are unable to show convergence due to the lack of regularity in the potentials as well as the lack of regularity due to the absence of the porous medium type self-diffusion. While the schemes developed in [14,19] are asymptotic preserving, their convergence to weak solutions of the respective equations could not be established. We reproduce the steady states of [19] that exhibit phase separation phenomena. Figure 6 displays the stationary state in the case $\nu=0.09$ and $\epsilon=0$. Even in the case of no regularising individual diffusion and with Newtonian cross-interactions we observe a numerical convergence order of one.

In the case of attractive-attractive cross-interactions, i.e. $W_{12}(x)=|x|=W_{21}(x)$, we observe an interesting phenomenon. Even in the absence of the individual diffusion, i.e. $\epsilon=0$, some additional mixing occurs even though we expect sharp boundaries, see Fig. 7, due to numerical diffusion. This is in contrast to the finite volume schemes proposed in $[14,19]$. 
Fig. 7 We choose $m_{1}=0.6$ and $m_{2}=0.1$ in order to be able to compare the stationary state with the explicit one given in [19].

We can see a strong resemblance between the numerical stationary state and the one obtained analytically. However there are some regimes of mixing due to numerical diffusion

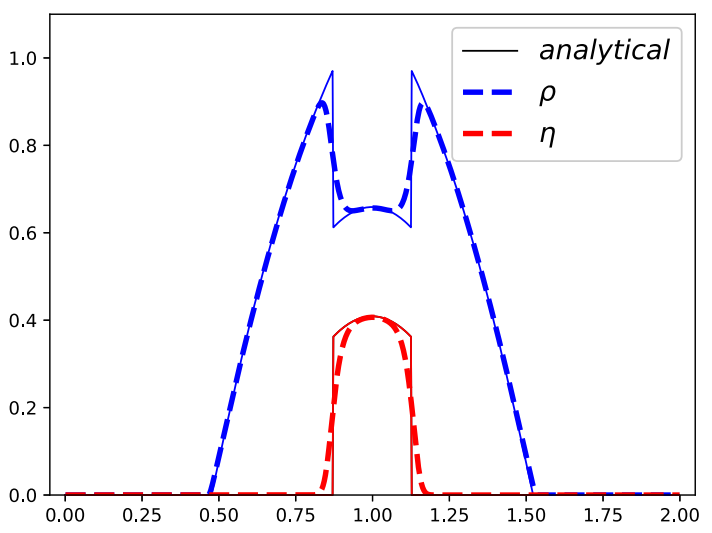

\subsection{Energy dissipation}

It is known that system (1) has a formal gradient flow structure, cf. [24], whenever $W_{12}=W_{21}$. In this case, the evolution of system 1 is such that it decays the energy functional

$$
\begin{aligned}
\mathfrak{E}(\rho, \eta): & =\frac{1}{2} \iint \rho W_{11} \star \rho+\eta W_{22} \eta \mathrm{d} x+\iint \rho W_{12} \star \eta \mathrm{d} x \\
& +\frac{1}{2} \int v(\rho+\eta)^{2}+\epsilon \rho^{2}+\epsilon \eta^{2} \mathrm{~d} x .
\end{aligned}
$$

Here, we present two examples, one corresponding to the potential

$$
W_{i i}(x)=\frac{x^{2}}{2}, \text { and } W_{i j}(x)=|x|,
$$

for $i, j=1,2$ and $i \neq j$, cf. Fig. 8, the other one corresponding to

$$
\begin{aligned}
& W_{i i}(x)=1-\exp \left(-\frac{(4 x)^{2}}{2}\right), \text { and } \\
& W_{i j}(x)=1+\exp \left(-(4 x)^{2}\right)-\exp \left(-\frac{2}{3}(4 x)^{2}\right),
\end{aligned}
$$

for $i, j=1,2$ and $i \neq j$, cf. Fig. 9 .

In the first case, we choose the initial data

$$
\rho(x)=c((x-2)(2.5-x))^{+}, \quad \text { and } \quad \eta(x)=c((x-1)(0.5-x))^{+},
$$

where $c>0$ normalises the mass to one. Due to the long-range we observe an attraction of the two initial bumps until they meet. They begin to mix until they are completely merged. The graph in the last panel shows the decay of the associated energy to a constant one which corresponds to the energy of the steady state. It appears the energy 


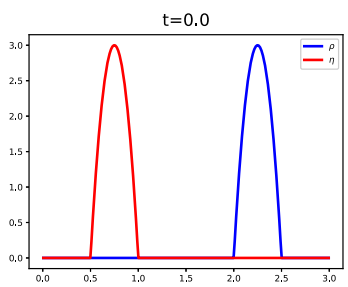

(a) $t=0$

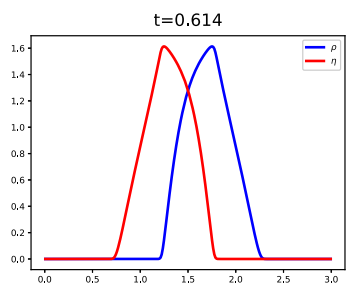

(d) $t=0.614$

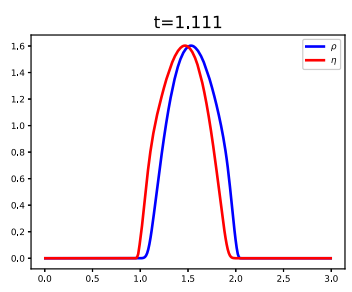

(g) $t=1.111$

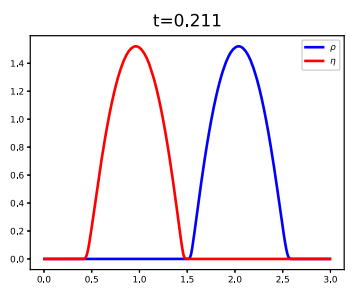

(b) $t=0.211$

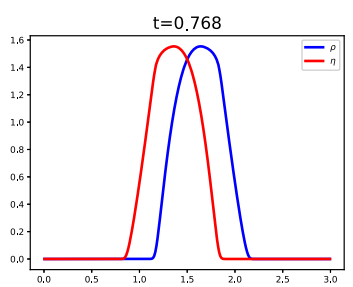

(e) $t=0.768$

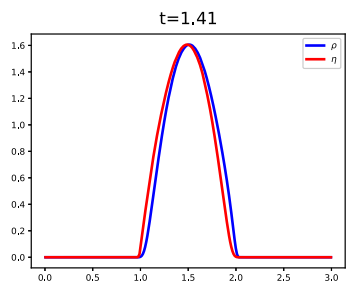

(h) $t=1.41$

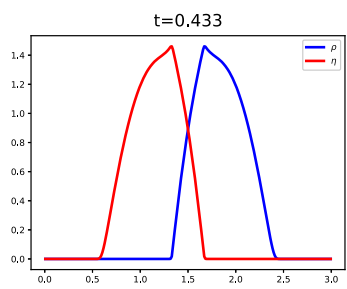

(c) $t=0.433$

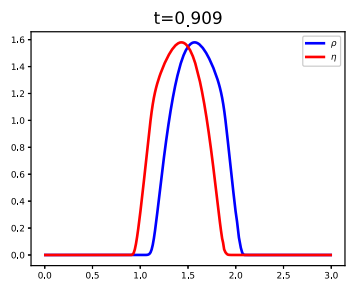

(f) $t=0.909$

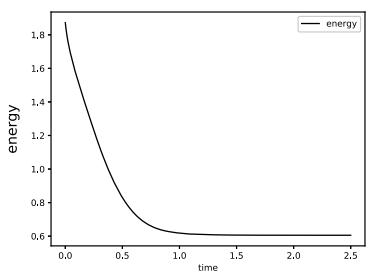

(i) Decay of the energy, $\mathfrak{E}(\rho, \eta)$.

Fig. 8 Evolution of segregated initial data for attractive-attractive interactions with corresponding potentials $W_{i i}(x)=x^{2} / 2$ and $W_{i j}(x)=|x|$. The two blobs move towards each other. The associated energy appears to converge exponentially fast to a constant while the profiles merge

is dissipated at an exponential rate but an analytic result for systems, corresponding to that of a single equation, cf. [21], is not known to our knowledge.

In the second case, for Gaussian potentials, we change the computational domain to $(0, \pi)$, for convenience. We choose the initial data

$$
\rho(x)=\sin (2 x)^{2}, \text { and } \cos (2 x)^{2},
$$

cf. Fig. 9. We observe the formation of nearly segregated clusters which, as the evolution continues, as begin to merge due to the nonlocal interaction. However, the short-range cross-interaction is working against this trend which explains that the evolution slows down just before the merging, a phenomenon which is also observed in meta-stability. After the 5 clusters have merged into three the profile stabilises which is reflected in the evolution of the energy, cf. graph in the panel. We still observe a decay, however, after a strong initial decrease the energy decays much slower for a while before going to the constant corresponding to the stationary state. The explanation lies in the increase of the internal energy. Initially, we have $\rho+\eta \equiv 1$ which is a minimiser of the internal energy. Due to the nonlocal interactions the system wants 


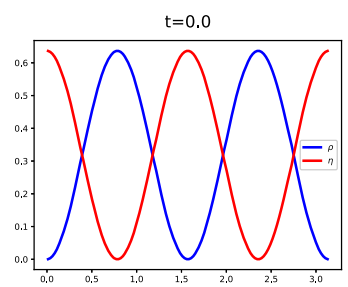

(a) $t=0.0$

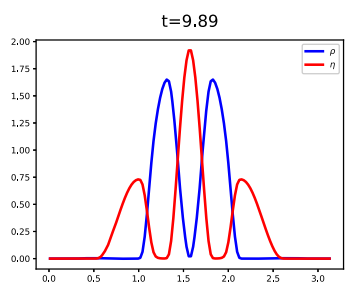

(d) $t=9.89$

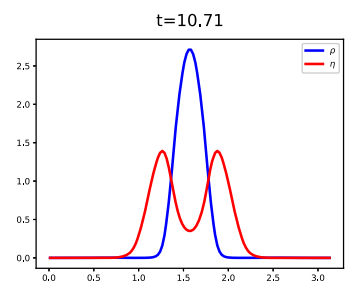

(g) $t=10.71$

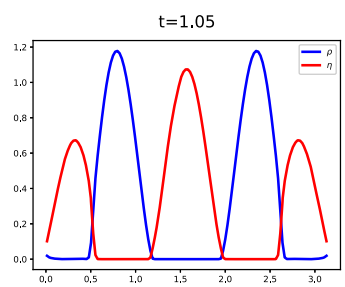

(b) $t=1.05$

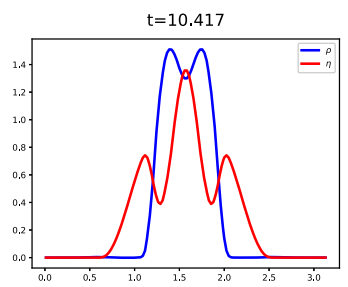

(e) $t=10.417$

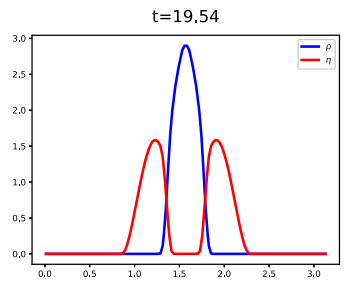

(h) $t=19.54$

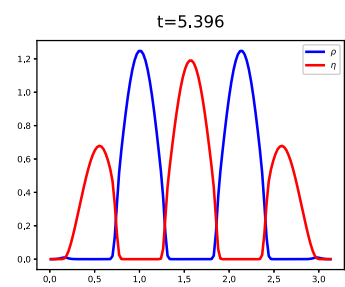

(c) $t=5.396$

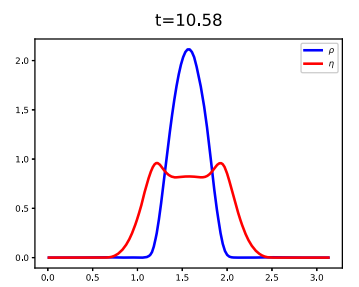

(f) $t=10.58$

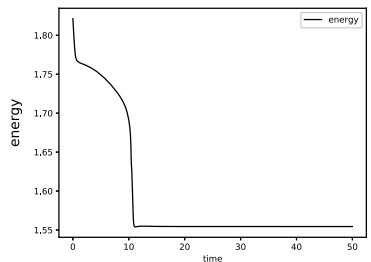

(i) Decay of the energy, $\mathfrak{E}(\rho, \eta)$.

Fig. 9 Evolution of mixed initial data, $\rho=\sin (2 x)^{2}$ and $\eta=\cos (2 x)^{2}$ on the domain $(0, \pi)$. The interactions are linear combinations of Gaussians modelling self-attraction whereas the cross-interactions are short-range repulsive and long-range attractive. The associated energy decays abruptly at the merging and separation between aggregates. The slower decay of the energy before $t \approx 10$ is due to the trade off between the local (internal) energy and the nonlocal interaction energy. After the rearrangement of the five initial clusters to only three, the energy stabilises

to rearrange but at the cost of increasing the internal energy to allow for a decrease in the interaction energy. This beautifully portrays the interplay of local and nonlocal effects. Similar effects are known in the context of meta-stability.

\section{Conclusion}

In this paper we presented a finite volume scheme for a system of non-local partial differential equations with cross-diffusion. We were able to reproduce a continuous energy estimate on the discrete level for our scheme. These discrete estimates for the approximate solution are enough to get compactness results and we are able to identify the limit of the approximate solutions as a weak solution of the equation. We complement the analytical part with numerical simulations. These back up our convergence result and we are also able to apply the scheme in cases in which we cannot 
show convergence. To this end we pushed the scheme to regimes of singular potentials also lacking the regularising porous medium type self-diffusion terms. Comparing them with the explicit stationary states from [19] we conclude the scheme performs well even in regimes it was not designed for.

Acknowledgements University of Oxford. JAC was partially supported by EPSRC Grant Number EP/P031587/1 and the Advanced Grant Nonlocal-CPD (Nonlocal PDEs for Complex Particle Dynamics: Phase Transitions, Patterns and Synchronization) of the European Research Council Executive Agency (ERC) under the European Union's Horizon 2020 research and innovation programme (Grant Agreement No. 883363).

Open Access This article is licensed under a Creative Commons Attribution 4.0 International License, which permits use, sharing, adaptation, distribution and reproduction in any medium or format, as long as you give appropriate credit to the original author(s) and the source, provide a link to the Creative Commons licence, and indicate if changes were made. The images or other third party material in this article are included in the article's Creative Commons licence, unless indicated otherwise in a credit line to the material. If material is not included in the article's Creative Commons licence and your intended use is not permitted by statutory regulation or exceeds the permitted use, you will need to obtain permission directly from the copyright holder. To view a copy of this licence, visit http://creativecommons.org/licenses/by/4.0/.

\section{References}

1. Attouch, H., Buttazzo, G., Michaille, G.: Variational Analysis in Sobolev and BV Spaces: Applications to PDEs and Optimization. SIAM, Philadelphia (2014)

2. Berendsen, J., Burger, M., Pietschmann, J.-F.: On a cross-diffusion model for multiple species with nonlocal interaction and size exclusion. Nonlinear Anal. 159, 10-39 (2017)

3. Bertsch, M., Dal Passo, R., Mimura, M.: A free boundary problem arising in a simplified tumour growth model of contact inhibition. Interfaces Free Bound. 12(2), 235-250 (2010)

4. Bertsch, M., Gurtin, M., Hilhorst, D.: On a degenerate diffusion equation of the form $c(z)_{t}=\varphi\left(z_{x}\right)_{x}$ with application to population dynamics. J. Differ. Equ. 67(1), 56-89 (1987)

5. Bertsch, M., Gurtin, M., Hilhorst, D., Peletier, L.: On interacting populations that disperse to avoid crowding: preservation of segregation. J. Math. Biol. 23(1), 1-13 (1985)

6. Bertsch, M., Gurtin, M., Hilhorst, D.: On interacting populations that disperse to avoid crowding: the case of equal dispersal velocities. Nonlinear Anal. Theory Methods Appl. 11(4), 493-499 (1987)

7. Bertsch, M., Hilhorst, D., Izuhara, H., Mimura, M.: A nonlinear parabolic-hyperbolic system for contact inhibition of cell-growth. Differ. Equ. Appl. 4(1), 137-157 (2012)

8. Bessemoulin-Chatard, M., Chainais-Hillairet, C., Filbet, F.: On discrete functional inequalities for some finite volume schemes. IMA J. Num. Anal. 35(3), 1125-1149 (2015)

9. Bessemoulin-Chatard, M., Filbet, F.: A finite volume scheme for nonlinear degenerate parabolic equations. SIAM J. Sci. Comput. 34(5), B559-B583 (2012)

10. Bruna, M., Burger, M., Ranetbauer, H., Wolfram, M.-T.: Cross-diffusion systems with excluded-volume effects and asymptotic gradient flow structures. J. Nonlinear Sci. 27(2), 687-719 (2017)

11. Burger, M., Di Francesco, M., Fagioli, S., Stevens, A.: Sorting phenomena in a mathematical model for two mutually attracting/repelling species. preprint arXiv:1704.04179 (2017)

12. Calvez, V., Carrillo, J.A.: Volume effects in the Keller-Segel model: energy estimates preventing blow-up. J. Math. Pures Appl. (9) 86(2), 155-175 (2006)

13. Carl, E.A.: Population control in arctic ground squirrels. Ecology 52(3), 395-413 (1971)

14. Carrillo, J.A., Chertock, A., Huang, Y.: A finite-volume method for nonlinear nonlocal equations with a gradient flow structure. Commun. Comput. Phys. 17(01), 233-258 (2015)

15. Carrillo, J.A., Choi, Y.-P., Hauray, M.: The derivation of swarming models: mean-field limit and Wasserstein distances. In: Muntean, A., Toschi, F. (eds.) Collective Dynamics from Bacteria to Crowds, pp. 1-46. Springer, Berlin (2014)

16. Carrillo, J.A., Colombi, A., Scianna, M.: Adhesion and volume constraints via nonlocal interactions lead to cell sorting. preprint arXiv:1706.08969 (2017) 
17. Carrillo, J.A., Fagioli, S., Santambrogio, F., Schmidtchen, M.: Splitting schemes \& segregation in reaction-(cross-)diffusion systems. arXiv preprint arXiv:1711.05434 (2017)

18. Carrillo, J.A., Huang, Y., Martin, S.: Explicit flock solutions for Quasi-Morse potentials. Eur. J. Appl. Math. 25(5), 553-578 (2014)

19. Carrillo, J.A., Huang, Y., Schmidtchen, M.: Zoology of a non-local cross-diffusion model for two species. arXiv preprint arXiv:1705.03320 (2017)

20. Carrillo, J.A., Martin, S., Panferov, V.: A new interaction potential for swarming models. Physica D 260, 112-126 (2013)

21. Carrillo, J.A., McCann, R.J., Villani, C., et al.: Kinetic equilibration rates for granular media and related equations: entropy dissipation and mass transportation estimates. Rev. Mat. Iberoam. 19(3), 971-1018 (2003)

22. Chainais-Hillairet, C., Filbet, F.: Asymptotic behaviour of a finite-volume scheme for the transient drift-diffusion model. IMA J. Numer. Anal. 27(4), 689-716 (2007)

23. Chen, X., Jüngel, A., Liu, J.-G.: A note on Aubin-Lions-Dubinskiı̆ lemmas. Acta Appl. Math. 133, 33-43 (2014)

24. Di Francesco, M., Esposito, A., Fagioli, S.: Nonlinear degenerate cross-diffusion systems with nonlocal interaction. Nonlinear Anal. 169, 94-117 (2018)

25. Di Francesco, M., Fagioli, S.: Measure solutions for non-local interaction pdes with two species. Nonlinearity 26(10), 2777 (2013)

26. Domschke, P., Trucu, D., Gerisch, A., Chaplain, M.A.J.: Mathematical modelling of cancer invasion: implications of cell adhesion variability for tumour infiltrative growth patterns. J. Theor. Biol. 361, 41-60 (2014)

27. D’Orsogna, M.R., Chuang, Y.-L., Bertozzi, A.L., Chayes, L.S.: Self-propelled particles with soft-core interactions: patterns, stability, and collapse. Phys. Rev. Lett. 96(10), 104302 (2006)

28. Eymard, R., Gallouët, T., Herbin, R.: Finite volume methods. Handb. Numer. Anal. 7, 713-1018 (2000)

29. Eymard, R., Gallouët, T., Herbin, R.: Finite volume methods. In: Ciarlet P.G., Lions, J.L. (eds.) Handbook of Numerical Analysis, vol. VII, pp. 713-1020. North-Holland, Amsterdam (2000). https://www. sciencedirect.com/handbook/handbook-of-numericalanalysis/vol/7/suppl/C

30. Filbet, F., Herda, M.: A finite volume scheme for boundary-driven convection-diffusion equations with relative entropy structure. Numer. Math. 137(3), 535-577 (2017)

31. Gerisch, A., Chaplain, M.A.J.: Mathematical modelling of cancer cell invasion of tissue: local and non-local models and the effect of adhesion. J. Theor. Biol. 250(4), 684-704 (2008)

32. Gurney, W., Nisbet, R.: The regulation of inhomogeneous populations. J. Theor. Biol. 52(2), 441-457 (1975)

33. Gurtin, M.E., Pipkin, A.: A note on interacting populations that disperse to avoid crowding. Q. Appl. Math. 42(1), 87-94 (1984)

34. Itô, Y.: The growth form of populations in some aphids, with special reference to the relation between population density and the movements. Res. Popul. Ecol. 1(1), 36-48 (1952)

35. Mogilner, A., Edelstein-Keshet, L.: A non-local model for a swarm. J. Math. Biol. 38(6), 534-570 (1999)

36. Morisita, M.: Population density and dispersal of a water strider Gerris lacustris: observations and considerations on animal aggregations. Contrib. Physiol. Ecol. Kyoto Univ. 65, 1-149 (1950)

37. Morisita, M.: Dispersion and population pressure: experimental studies on the population density of an ant-lion, Glenuroides japonicus m'1 (2). Jpn. J. Ecol. 4(71), 9 (1954)

38. Murakawa, H., Togashi, H.: Continuous models for cell-cell adhesion. J. Theor. Biol. 374, 1-12 (2015)

39. Painter, K.J., Bloomfield, J.M., Sherratt, J.A., Gerisch, A.: A nonlocal model for contact attraction and repulsion in heterogeneous cell populations. Bull. Math. Biol. 77(6), 1132-1165 (2015)

40. Painter, K.J., Hillen, T.: Volume-filling and quorum-sensing in models for chemosensitive movement. Can. Appl. Math. Q. 10(4), 501-543 (2002)

41. Topaz, C.M., Bertozzi, A.L., Lewis, M.A.: A nonlocal continuum model for biological aggregation. Bull. Math. Biol. 68(7), 1601-1623 (2006)

42. Volkening, A., Sandstede, B.: Modelling stripe formation in zebrafish: an agent-based approach. J. R. Soc. Interface (2015). https://doi.org/10.1098/rsif.2015.0812

Publisher's Note Springer Nature remains neutral with regard to jurisdictional claims in published maps and institutional affiliations. 